\title{
Evolution of Nuclear Receptors in Platyhelminths
}

Departments of Biochemistry and Structural Biology and Pathology and Laboratory Medicine,

4 University of Texas Health Sciences Center, San Antonio, Texas, 78229-3800, USA

${ }^{1}$ Present Address: Canget BioTekpharma LLC, NY State Center of Excellence in Bioinformatics

6 \& Life Sciences, 701 Ellicott Street, Buffalo, NY 14203, USA

$7 \quad{ }^{*}$ Corresponding author: loverde@uthscsa.edu

8 Short Title: Platyhelminth Nuclear Receptors

Keywords: nuclear receptors, gene duplication, evolution, Platyhelminths

Abbreviation: 2DBD-NR: nuclear receptor with two DBDs; AR, androgen receptor; CAR, constitutive androstane receptor; COUP-TF, chicken ovalbumin upstream promoter transcription factor; DBD, DNA binding domain; DSF, Drosophila dissatisfaction gene; E75, Drosophila ecdysone-induced protein 75; E78, Drosophila ecdysone-induced protein 78; EAR, V-erbArelated gene; EGON, embryonic gonad protein; ER, estrogen receptor; ERR, estrogen related receptor; FTZ-F1, fushi tarazu-factor 1; FXR, farnesoid X receptor; GCNF, germ cell nuclear factor; GR, glucuronide receptor; HNF-4, hepatocyte nuclear factor 4; HR96, Drosophila 17 hormone receptor 96; LBD, ligand binding domain; LRH-1, liver receptor homologue 1; LXR, liver X receptor; MR, mineralocorticoid receptor; NGFI-B, nerve growth factor-induced B; NHR236, nematode nuclear receptor 236; NR, Nuclear receptor; Nurr1, nuclear receptor related 1; NOR1, neuron-derived orphan receptor 1; PNR, photoreceptor-specific nuclear receptor; PR,

21 progesterone receptor; PXR, pregnane X receptor; PPAR, peroxisome proliferators-activated 22 receptor; Rev-erb, nuclear receptor-related protein coded on the opposite strand of the thyroid 23 hormone receptor gene; RAR, retinoic acid receptor; ROR, retinoid-related orphan receptor; $\mathbf{R X R}$, 
bioRxiv preprint doi: https://doi.org/10.1101/2021.04.14.439782; this version posted April 14, 2021. The copyright holder for this preprint (which

was not certified by peer review) is the author/funder, who has granted bioRxiv a license to display the preprint in perpetuity. It is made available under aCC-BY 4.0 International license.

24 retinoid $\mathrm{X}$ receptor; SF1, Steroidogenic factor 1; SR, steroid receptor; SVP, seven up;

25 Supplementary nuclear receptors, SupNRs, supplementary nuclear receptors; TLL, Drosophila

26 tailless gene; TLX, homologue of the Drosophila tailless gene; TR, thyroid hormone receptor;

27 TR2/4, testicular receptor 2/4; USP, ultraspiracle; VDR, vitamin D receptor. 


\section{ABSTRACT}

Since the first complete set of Platyhelminth nuclear receptors (NRs) from Schistosoma mansoni were identified a decade ago, more flatworm genome data is available to identify their

32 NR complement and to analyze the evolutionary relationship of Platyhelminth NRs. NRs are important transcriptional modulators that regulate development, differentiation and reproduction of animals. In this study, NRs are identified in genome databases of thirty-three species including in all Platyhelminth classes (Rhabditophora, Monogenea, Cestoda and Trematoda). Phylogenetic analysis shows that NRs in Platyhelminths follow two different evolutionary lineages: 1) NRs in

37 a free-living freshwater flatworm (Schmidtea mediterranea) and all parasitic flatworms share the same evolutionary lineage with extensive gene loss. 2) NRs in a free-living intertidal zone flatworm (Macrostomum lignano) follow a different evolutionary lineage with a feature of multiple gene duplication and gene divergence. The DNA binding domain (DBD) is the most conserved region in NRs which contains two C4-type zinc finger motifs. A novel zinc finger motif is identified in parasitic flatworm NRs: the second zinc finger of parasitic Platyhelminth HR96b possesses a CHC2 motif which is not found in NRs of all other animals. In this study, novel NRs (members of NR subfamily 3 and 6) are identified in flatworms, this result demonstrates that members of all six classical NR subfamilies are present in the Platyhelminth phylum. NR gene duplication, loss and divergence in Platyhelminths are analyzed along with the evolutionary relationship of Platyhelminth NRs. 


\section{2}

53

54

55

56

57

\section{INTRODUCTION}

Platyhelminths (flatworms) are one of the largest animal phyla, it includes more than 20,000 species $[1,2]$. Some flatworms are important disease-causing parasites of humans and livestock, e.g. Schistosomiasis, Paragonimiasis and Cestodiasis. Platyhelminths are bilaterally symmetrical, non-segmented acoelomates without an anus. Although they have an excretory system they lack respiratory and circulatory systems, all flatworms are hermaphroditic except Schistosoma sp. where asexual and sexual reproduction are present [3]. Platyhelminths are traditionally divided into four classes: Rhabditophora, Monogenea, Cestoda (tapeworms) and Trematoda (flukes). The class Rhabditophora includes all free-living flatworms, while all members in classes of Monogenea, Trematoda, and Cestoda are parasitic flatworms. From an evolutionary point of view, the parasitic classes arose from a primitive free-living flatworm [3]. Nuclear receptors (NRs) are important transcriptional modulators that regulate development, differentiation and reproduction of animals. Most of NRs share a common tertiary structure: A/B-C-D-E domains. The A/B domain is highly variable, the $\mathrm{C}$ domain is the DNAbinding domain (DBD) which is the most conserved region containing two zinc finger motifs, the $\mathrm{D}$ domain a flexible hinge between the $\mathrm{C}$ and $\mathrm{E}$ domains and is poorly conserved, the $\mathrm{E}$ domain contains the ligand binding domain (LBD) that is involved in transcriptional activation. Atypical NRs are found in some animals, e.g. NRs with a DBD but no LBD are found in arthropods and nematodes, members without a DBD but with a LBD are present in some vertebrates, and NRs with two DBDs and a single LBD (2DBD-NRs) are identified in protostomes. A phylogenetic analysis of the NRs divides them into six classical subfamilies by alignment of the conserved DBD [4]. The early study of the complete genome sequence of ecdysozoan Arthropods (Drosophila melanogaster) [5] the mosquito (Anopheles gambiae) [6], free-living nematodes (Caenorhabditis 
75 elegans and C. briggsae) [7, 8], tunicata (Ciona intestinalis) [9], mammalians: rat (Rattus norvegicus), mouse (Mus musculus) [10] and human (Homo sapiens) [11]) revealed that NRs in

77 insects, tunicata and mammalians share the same evolutionary lineage with extensive gene

78 loss/duplication, while NRs in nematodes follow a different evolutionary lineage with a feature of

79 multiple duplication of SupNRs and gene loss.

Since we identified the first complete set of Platyhelminths nuclear receptors (NRs) from

81 Schistosoma mansoni a decade ago [12], more flatworm genome data has become available to

82 identify their NR complement. Analysis of the NR complement of the blood fluke Schistosoma mansoni [12-14] and tapeworm Echinococcus multilocularis [13-15] shows that NRs in S. mansoni and E. multilocularis share the same evolutionary lineage as that of Deuterostomia and the

85 arthropods in the Ecdysozoan clade of the Protostomia, but some divergent NRs in flatworms are not present in Deuterostomia or/and arthropods, for example 2DBD-NRs [16]. In this study, we

87 analyzed genome databases of Platyhelminth Wormbase website including species from all of the four classes in Platyhelminths [17, 18]. Identification of the NR complement will contribute to a better understanding of the evolution of NRs in Platyhelminths.

\section{MATERIALS AND METHODS}

\section{Data mining}

Nuclear receptors in Platyhelminths were mined from the genome databases in Wormbase 
position (JP) and GT-AG rule if there was an intron in the DBD coding region. The analyzed

99

100

101

102

103

104

105

106

107

108

109

110

111

112

113

114

115

116

117

118

Platyhelminth genome databases are listed in Table 1.

\section{Phylogenetic analysis}

Phylogenetic trees were constructed from deduced amino acid sequences of the DBD and/or ligand binding domain (LBD), the sequences are aligned with ClustalW [19], phylogenetic analysis of the data set is carried out using Bayesian inference (MrBAYES v3.1.1) [20] with a mix amino acid replacement model + gamma rates. The trees were started randomly; four simultaneous Markov chains were run for 5 million generations, the trees were sampled every 100 generations, the Bayesian posterior probabilities (PPs) were calculated using a Markov chain Monte Carlo (MCMC) sampling approach implemented in MrBAYES v3.1.1, with a burn-in value setting at 12,500. The same data set was also tested by Maximum Likelihood method under LG substitution model with a gamma distribution of rates between sites (eight categories, parameter alpha, estimated by the program) using PhyML 3.0 [21] with support values obtained by bootstrapping a 1000 replicates. For GenBank accession numbers of published NR sequences used in this study see S1 file.

\section{RESULTS}

Genome databases of 33 species of Platyhelminths from Worm database were mined. The analyzed flatworm species include two species from class Rhabditophora, two species from class Monogenea, fifteen species from class Cestoda and fourteen species from class Trematoda (Table 1). For amino acid sequences of the DBD identified in these species see $\mathbf{S 2}$ file. 
bioRxiv preprint doi: https://doi.org/10.1101/2021.04.14.439782; this version posted April 14, 2021. The copyright holder for this preprint (which was not certified by peer review) is the author/funder, who has granted bioRxiv a license to display the preprint in perpetuity. It is made available under aCC-BY 4.0 International license.

Table 1. Nuclear receptors analyzed in Platyhelminth species

\begin{tabular}{|c|c|c|c|c|}
\hline CLASS & ORDER & FAMILY & $\begin{array}{c}\text { SPECIES } \\
\text { REFERENCES }\end{array}$ & SHOR DESCRIPTION \\
\hline \multirow[t]{2}{*}{ Rhabditophora } & Rhabditophora & Rhabditophorae & $\begin{array}{l}\text { Macrostomum lignano (Ml) [22, } \\
23]\end{array}$ & $\begin{array}{l}\text { Free-living, intertidal flatworm that can regenerate most of its } \\
\text { body parts. }\end{array}$ \\
\hline & Tricladida & Dugesidae & $\begin{array}{l}\text { Schmidtea mediterranea (Sme) } \\
{[24-26]} \\
\end{array}$ & $\begin{array}{l}\text { Free-living, freshwater flatworm that can regenerate an entire } \\
\text { organism. }\end{array}$ \\
\hline \multirow[t]{2}{*}{ Monogenea } & Monopisthocotylea & Gyrodactylidae & Gyrodactylus salaris (Gs) [27] & Salmon fluke that lives on the body surface of freshwater fish. \\
\hline & Polyopisthocotylea & Polystomatidae & $\begin{array}{c}\text { Protopolystoma xenopodis }(P x) \\
{[28]}\end{array}$ & $\begin{array}{l}\text { Fluke of African clawed frogs where the adult worms live in } \\
\text { the host's urinary bladder. }\end{array}$ \\
\hline \multirow[t]{15}{*}{ Cestoda } & Cyclophyllidea & Hymenolepididae & Hymenolepis diminuta (Hd) [28] & $\begin{array}{l}\text { Rat tapeworm. The intermediate hosts: arthropod; the } \\
\text { definitive hosts: rodent. }\end{array}$ \\
\hline & & & H. microstoma (Hm) [29] & $\begin{array}{l}\text { Rodent tapeworm. The intermediate hosts: arthropod; the } \\
\text { definitive hosts: rodent. }\end{array}$ \\
\hline & & & H. nana (Hn) [28] & $\begin{array}{l}\text { Rodent tapeworm. The intermediate hosts: arthropod; the } \\
\text { definitive hosts: human and rodent. }\end{array}$ \\
\hline & & Taeniidae & $\begin{array}{c}\text { Echinococcus Canadensis }(E c) \\
{[30]}\end{array}$ & $\begin{array}{l}\text { Canid tapeworm. The intermediate hosts: cervid, camels, cattle } \\
\text { etc.; the definitive host: dogs and other canids. }\end{array}$ \\
\hline & & & E. granulosus (Eg) $[29,31]$ & $\begin{array}{l}\text { Dog tapeworm. The intermediate hosts: human, horse; the } \\
\text { definitive host: dogs. }\end{array}$ \\
\hline & & & E. multilocularis (Em) [29] & $\begin{array}{l}\text { Fox tapeworm. The intermediate hosts: wild rodents; the } \\
\text { definitive hosts: foxes, coyotes, dogs and other canids. }\end{array}$ \\
\hline & & & Taenia asiatica (Ta) [32] & $\begin{array}{l}\text { Human tapeworm. The intermediate hosts: pigs; the definitive } \\
\text { host: human. }\end{array}$ \\
\hline & & & T. multiceps (Tm) [33] & $\begin{array}{l}\text { Dog tapeworm. The intermediate hosts: sheep, goat and cattle; } \\
\text { the definitive hosts: Canids. }\end{array}$ \\
\hline & & & T. saginata (Ts) [32] & $\begin{array}{l}\text { Beef tapeworm. The intermediate hosts: Cattle; the definitive } \\
\text { hosts: human. }\end{array}$ \\
\hline & & & T. solium (Tso) [29] & $\begin{array}{l}\text { Pork tapeworm. The intermediate host: pigs; the definitive } \\
\text { host: human. }\end{array}$ \\
\hline & & & $\begin{array}{c}\text { Hydatigera taeniaeformis }(H t) \\
{[28]}\end{array}$ & $\begin{array}{l}\text { Cat tapeworm. The intermediate hosts: rodents; the definitive } \\
\text { hosts: cats. }\end{array}$ \\
\hline & & Mesocestoididae & Mesocestoides corti (Mc) [28] & $\begin{array}{l}\text { Mouse tapeworm. The intermediate host: birds and small } \\
\text { mammals; the definitive host: cats and dogs. }\end{array}$ \\
\hline & Diphyllobotridea & Diphyllobothriidae & 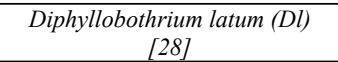 & $\begin{array}{l}\text { Human tapeworm. The intermediate hosts: fish; the definitive } \\
\text { hosts: human. }\end{array}$ \\
\hline & & & $\begin{array}{l}\text { Schistocephalus solidus (Ss) } \\
{\left[\begin{array}{l}\text { [28] }\end{array}\right.}\end{array}$ & $\begin{array}{l}\text { Bird tapeworm. The first intermediate hosts: cyclopoid } \\
\text { copepod; the second intermediate hosts: fish; the definitive } \\
\text { hosts: fish-eating water bird. }\end{array}$ \\
\hline & & & $\begin{array}{l}\text { Spirometra erinaceieuropaei } \\
\text { (Se) [34] }\end{array}$ & $\begin{array}{l}\text { Tapeworm. The first intermediate hosts: cyclopoid copepod; } \\
\text { the second intermediate hosts: fish, reptiles, or other } \\
\text { amphibians; the definitive hosts: cats and dogs. }\end{array}$ \\
\hline \multirow[t]{14}{*}{ Trematoda } & Opistorchida & Opistorchiidae & Clonorchis sinensis (Cs) [35] & $\begin{array}{l}\text { Human liver fluke. The first intermediate hosts: freshwater } \\
\text { snails; the second intermediate hosts: freshwater fish; the } \\
\text { definitive hosts: human and other mammals. }\end{array}$ \\
\hline & & & Opisthorchis felineus $(O f)[36]$ & $\begin{array}{l}\text { Cat liver fluke. The first intermediate hosts: freshwater snails; } \\
\text { the second intermediate hosts: freshwater fish; the definitive } \\
\text { hosts: human and other mammals. }\end{array}$ \\
\hline & & & O. viverrini $(O v)[37]$ & $\begin{array}{l}\text { Human liver fluke. The first intermediate hosts: freshwater } \\
\text { snails; the second intermediate hosts: freshwater fish; the } \\
\text { definitive hosts: human and other mammals. }\end{array}$ \\
\hline & Echinostomida & Echinostomatidae & Echinostoma caproni (Eca) [28] & $\begin{array}{l}\text { Intestinal fluke. The first intermediate hosts: freshwater snails; } \\
\text { the second intermediate hosts: freshwater snails or frogs. } \\
\text { Humans can be the definitive host. }\end{array}$ \\
\hline & & Fasciolidae & Fasciola hepatica $(F h)[38,39]$ & $\begin{array}{l}\text { Liver fluke. The intermediate hosts: freshwater snails; The } \\
\text { definitive hosts: cattle, sheep and other mammals including } \\
\text { human. }\end{array}$ \\
\hline & Strigeidida & Schistosomatidae & Schistosoma bovis (Sb) [40] & $\begin{array}{l}\text { Blood fluke. The intermediate hosts: freshwater snails; the } \\
\text { definitive host: ruminants (cattle, goats, sheep, etc). }\end{array}$ \\
\hline & & & S. curassoni (Sc) [28] & $\begin{array}{l}\text { Blood fluke. The intermediate hosts: freshwater snails; the } \\
\text { definitive hosts: ruminants (cattle, goat, sheep, etc). }\end{array}$ \\
\hline & & & S. haematobium (Sh) [41] & $\begin{array}{l}\text { Blood fluke. The intermediate hosts: freshwater snails; the } \\
\text { definitive hosts: cattle, goat or sheep. }\end{array}$ \\
\hline & & & S. japonicum (Sj) [42] & $\begin{array}{l}\text { HZoonotic blood fluke. The intermediate hosts: freshwater } \\
\text { snails; the definitive hosts: human. }\end{array}$ \\
\hline & & & S. mansoni (Sm) [43] & $\begin{array}{l}\text { Human blood fluke. The intermediate hosts: freshwater snails; } \\
\text { the definitive hosts: human. }\end{array}$ \\
\hline & & & S. margrebowiei (Sma) [28] & $\begin{array}{l}\text { Blood fluke. The intermediate hosts: freshwater snails; the } \\
\text { definitive hosts: antelope, buffalo and waterbuck. }\end{array}$ \\
\hline & & & S. mattheei (Sma) [28] & $\begin{array}{l}\text { Blood fluke. The intermediate hosts: freshwater snails; the } \\
\text { definitive hosts: bovid. }\end{array}$ \\
\hline & & & S. rodhaini (Sr) [28] & $\begin{array}{l}\text { Blood fluke. The intermediate hosts: freshwater snails; the } \\
\text { definitive host: rodent. }\end{array}$ \\
\hline & & & $\begin{array}{c}\text { Trichobilharzia regenti }(\mathrm{Tr}) \\
{[28]}\end{array}$ & $\begin{array}{l}\text { Neuropathogenic fluke. The intermediate host: freshwater } \\
\text { snail; the definitive host: avian. }\end{array}$ \\
\hline
\end{tabular}




\section{NRs in Platyhelminths}

\subsection{Class: Monogenea}

Most of the species in this class are ectoparasitic that live on the gills or skin of freshwater and marine fishes. Genome database of two species of Monogenea (Protopolystoma xenopodis [28] and Gyrodactylus salaris [27] ) were analyzed.

\subsubsection{Order: Polyopisthocotylea}

Protopolystoma xenopodis of Polystomatidae family was analyzed. P. xenopodis is a fluke of African clawed frogs its adult worms lives in the urinary bladder of the host. Twenty-three NRs were identified in P. xenopodis. Phylogenetic analysis shows they belong to five different NR subfamilies: seven members are from subfamily 1 (TR, E78, three HR96s and two divergent members), ten members are from subfamily 2 (HNF4, two RXRs, TR4, TLL, PNR, DSF, two Coup-TFs and NHR236), one member is from subfamily 4 (NR4A), two members are from subfamily 5 (FTZ-F1 and HR39) and three members belong to 2DBD-NRs (Table 2, S1 Fig).

One interesting result is that two divergent NRs from subfamily 1 (PxNR1a and PxNR1b) are present in P. xenopodis. Phylogenetic analysis shows that PxNR1a is an orthologue of Schistosoma mansoni NR1 (SmNR1) [44] (S1 Fig) and PxNR1b is an orthologue of the Cestode Echinococcus granulosus HR3 (EgHR3) [45]. Further analysis shows that the orthologue of PxNR1b is present in all analyzed tapeworms and also exists in the free-living Platyhelminth Schmidtea mediterranea (nhr10, [46]). Phylogenetic analysis of the DBD sequences shows that Platyhelminth NR1b group clustered together with Drosophila HR3/human ROR/Mollusca HR3 group but is outside of the HR3/ROR group (S2 Fig). To further demonstrate whether PxNR1b is an orthologue of ROR, DBD with LBD sequences or only LBD sequence of PxNR1b orthologue (EgHR3 and SmeNR1b) were analyzed. It turns out that the LBD sequence of PxNR1b is not 
bioRxiv preprint doi: https://doi.org/10.1101/2021.04.14.439782; this version posted April 14, 2021. The copyright holder for this preprint (which was not certified by peer review) is the author/funder, who has granted bioRxiv a license to display the preprint in perpetuity. It is made available under aCC-BY 4.0 International license.

\section{Table. 2. NRs in Platyhelminths}

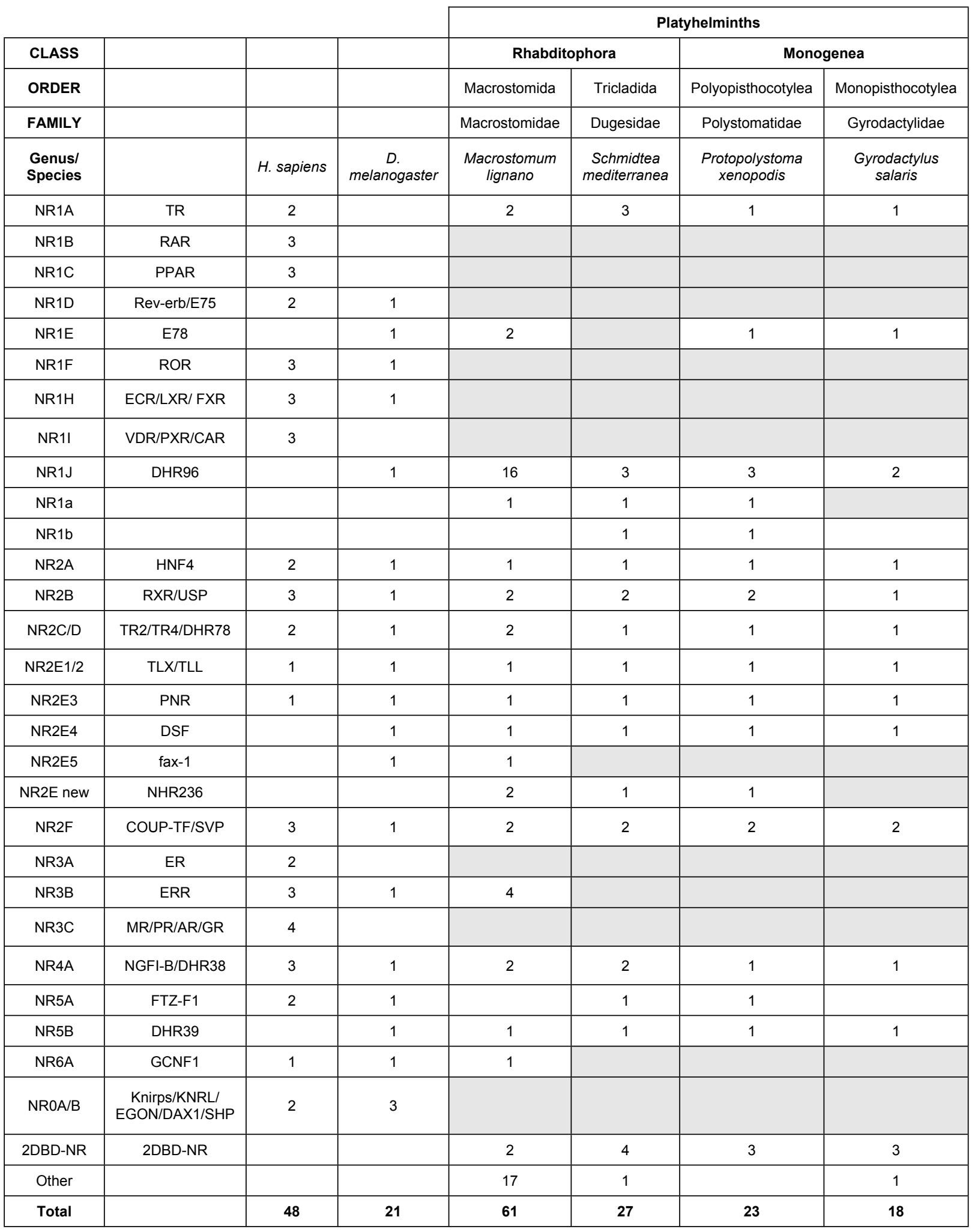


bioRxiv preprint doi: https://doi.org/10.1101/2021.04.14.439782; thiBlaglsimiptrated April 14, 2021. The copyright holder for this preprint (which was not certified by peer review) is the author/funder, who has granted bioRxiv a license to display the preprint in perpetuity. It is made

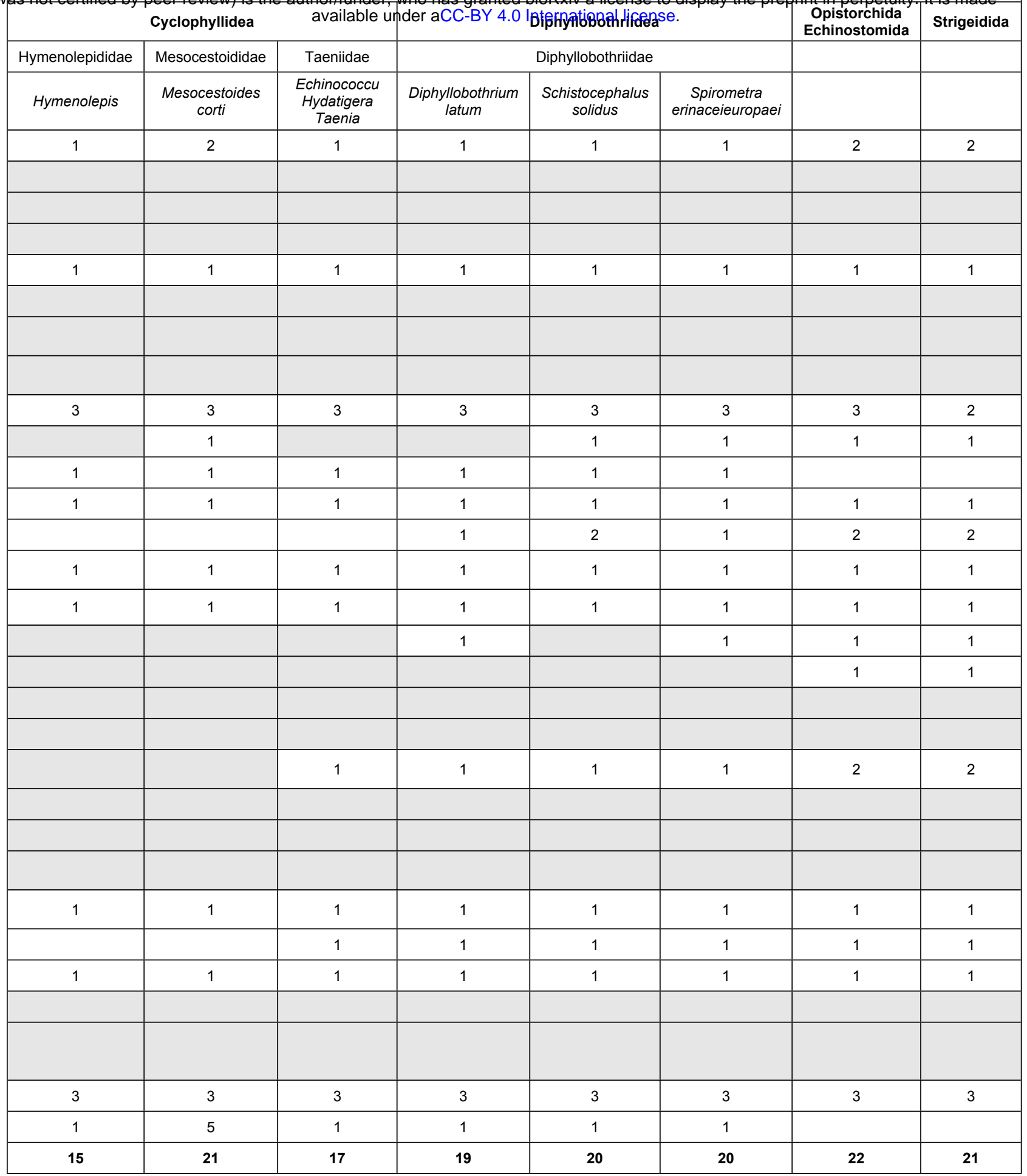

148 available. Phylogenetic analysis results show that EgHR3 and SmeNR1b are clustered with the

149 E75 group if both DBD and LBD sequences are analyzed, but they are clustered with E78 group 
if only LBD sequences are analyzed. These results suggest that PxNR1b orthologues are a group of divergent NRs that were present in a common Platyhelminth ancestor (S3 - S4 Figs).

Previously, we demonstrated that a novel NR2E member (orthologue of nematode Caenorhabditis elegans NHR236) was present in Cnidaria, Arthropoda, free-living Platyhelminths, Mollusca and Echinodermata [14]. In this study, an orthologue of NHR236 was identified in $P$. xenopodis (Table 2, S1 Fig). Phylogenetic analysis shows that NHR236 orthologues have a unique P-box sequence (CDGCRG) and they form a new NR subgroup in NR2E super group and its progenitor gene was present in a common ancestor of Porifera and Bilateral [14]. It is the first time that an orthologue of NHR236 has been shown to exist in parasitic Platyhelminths.

\subsubsection{Order Monopisthocotylea}

Gyrodactylus salaris of Gyrodactylidae family was analyzed. G. salaris is a fish fluke which lives on the body surface of freshwater fish. G. salaris belongs to family Gyrodactylidae of order Monopisthocotylea. Eighteen NRs from four NR subfamilies were identified in G. salaris. They include four members from subfamily 1 (TR, E78 and two HR96s), eight members from subfamily 2 (HNF4, RXR, TR4, TLL, PNR, DSF and two Coup-TFs), one member from subfamily 4 (NR4A), one member from subfamily 5 (HR39), three 2DND-NRs and one divergent NR which has an unknown P-box sequence (EPCKV) (Table 2, S5 Fig). A comparison of the NR complement with that of P. xenopodis, shows there are three HR96s in P. xenopodis but only two in G. salaris; two RXRs are present in P. xenopodis but only one in G. salaris; two NR1 divergent members (PxNR1a and PxNR1b) and a NHR236 orthologue identified in P. xenopodis that is missing in G. salaris. A G. salaris divergent NR is not found in P. xenopodis (Table 2).

\subsection{Class Cestoda}

\subsubsection{Order: Cyclophyllidea}




\subsubsection{Hymenolepididae family}

Three species in the genus Hymenolepis (H. diminuta, H. microstoma and H. nana) were analyzed. They are all rodent tapeworms and their intermediate hosts are arthropods. Fifteen NRs were identified in $H$. diminuta and $H$. microstoma. They include six members from subfamily 1 (TR, E78, three HR96s and NR1b), three NRs from subfamily 2 (HNF4, TR4 and TLL), one member from subfamily 4 (NR4A), one member from subfamily 5 (HR39), three 2DBD-NRs and one divergent NR. Fourteen NRs were identified in $H$. nana, a divergent NR identified in $H$. diminuta and H. microstoma is not present in H. nana (Table 2, S2 file and S6 Fig).

\subsubsection{Mesocestoididae family}

NR complement of Mesocestoides corti was mined and analyzed. M. corti is a mouse tapeworm, its intermediate hosts are birds and small mammals; its definitive host can be cats and dogs. Twenty-one NRs were identified in $M$. corti. There were eight members from subfamily 1 (two TRs, E78, three HR96s, NR1a and NR1b); three members from subfamily 2 (NHF4, TR4 and TLL); one member from subfamily 4 (NR4A); one member from subfamily 5 (HR39); three 2DND-NRs and five divergent NRs (Table 2, S2 file, S7 Fig).

\subsubsection{Taeniidae family}

NR complement in Taeniidae has been reported from the fox tapeworm Echinococcus multilocularis where seventeen NRs were identified [15]. In this study, we expanded the analysis

to include three genera (Echinococcus, Taenia and Hydatigera), they are 1) E. canadensis (a canid tapeworm its intermediate hosts are cervid, camels and cattle and the definitive hosts are dogs and other canids). 2) E. granulosus (a dog tapeworm where intermediate hosts are humans and horses. The definitive hosts are dogs). 3) E. multilocularis (a fox tapeworm. Its intermediate hosts are rodents and the definitive hosts are foxes, coyotes, dogs and other canids). 4) T. asiatica (a human 
tapeworm whose intermediate hosts are pigs). 5) T. multiceps (a dog tapeworm whose intermediate

197

198

199

200

201

202

203

204

205

206

207

208

209

210

211

212

213

214

215

216

217

hosts are sheep, goats and cattle). 6) T. solium (the pork tapeworm its intermediate hosts are pigs and the definitive hosts are humans). 7) H. taeniaeformis (a cat tapeworm its intermediate hosts are rodents).

Phylogenetic analysis result shows that all of above species share the same NR complement as E. multilocularis [15]; they exhibit six members from subfamily 1 (TR, E78, three HR96s and NR1b); four members from subfamily 2 (NHF4, TR4, TLL and Coup-TFI), one member from subfamily 4 (NR4A), two members from subfamily 5 (FTZ-F1and HR39), three 2DBD-NRs and a divergent NR. The divergent NR has a P-box of CDSCRA that is a homologue to SmRXR2 in the LBD [15] (Table 2, S8, S9, S10 Figs).

\subsubsection{Order: Diphyllobothriidea}

Three species from three genera of Diphyllobothriidae family were analyzed. They are 1) Dibothriocephalus latus (a human tapeworm its intermediate hosts are fish). 2) Schitocephalus solidus (a bird tapeworm). Its first intermediate hosts are cyclopoid and the second intermediate hosts are fish. Its definitive hosts are fish-eating water birds). 3) Spirometra erinaceieuropaei (a tapeworm its first intermediate hosts are cyclopoids and the second intermediate hosts are fish, reptiles, or other amphibians. Its definitive hosts are cats and dogs) (Table 1).

Nineteen NRs were identified in D. latus including six members from subfamily 1 (TR, E78, three HR96s and NR1b); five members from subfamily 2 (HNF4, RXR, TR4, TLL, PNR and Coup-TF); one member from subfamily 4 (NR4A); two members from subfamily 5 (FTZ-F1 and HR96), three 2DBD-NRs and one divergent NRs which is similar to Coup-TF with a different pbox sequence (Table 2). 
Twenty NRs were identified in S. solidus including seven members from subfamily 1 (TR, E78, three HR96s, NR1a and NR1b); six members from subfamily 2 (HNF4, two RXRs, TR4, TLL and Coup-TF), one member from subfamily 4 (NR4A), two members from subfamily 5 members (FTZ-F1a and FTZ-F1b), three 2DBD-NRs and one divergent NRs which is an orthologue of D. latus divergent NR (Table 2, S11 Fig).

Twenty NRs were identified in S. erinaceieuropaei. They include seven members from subfamily 1 (TR, E78, three HR96s, NR1a and NR1b); six members from subfamily 2 (HNF4, RXR, TR4, TLL, PNR and Coup-TF); one member from subfamily 4 (NR4A); two members from subfamily 5 members (FTZ-F1a and FTZ-F1b); three 2DBD-NRs and one divergent NRs which is an orthologue of D. latus and S. solidus divergent NR (Table 2, S12 Fig). orders, RXR and PNR are present in Diphyllobothriidae but they are missing in Cyclophyllidea.

\subsection{Class Trematoda}

\subsubsection{Order: Strigeidida}

NR complements in nine species of Schistosomatidae family were analyzed, they are 1)

237 Schistosoma mansoni (a human blood fluke its intermediate hosts are freshwater snails). 2) $S$.

238 haematobium (a human blood fluke its intermediate hosts are freshwater snails). 3) S. japonicum

239 (a zoonotic blood fluke, its intermediate hosts are amphibious snails). 4) S. curassoni (a blood 
goat, sheep). 5) S. margrebowiei (a blood fluke, its intermediate hosts are freshwater snails and the definitive hosts are antelope, buffalo and waterbuck). 6) S. mattheei (a blood fluke, its intermediate hosts are freshwater snails and the definitive hosts are bovids). 7) S. rodhaini (a blood fluke, its intermediate hosts are freshwater snails and the definitive host are rodents). 8) S. bovis (a blood fluke, its intermediate hosts are freshwater snails and the definitive hosts are ruminants (cattle, goats, sheep)). 9) Trichobilharzia regent (a neuropathogenic fluke, its intermediate host are freshwater snails and the definitive hosts are avian species).

NRs in parasitic trematodes were well described in $S$. mansoni [12-14, 16, 44, 47-62] . Twenty-one NRs were identified in $S$. mansoni including seven members from subfamily 1 (two TRs, E78, two DHR96s, NR1a (SmNR1 orthologue); nine members from subfamily 2 (HNF4, two RXRs, TR4, TLL, PNR, DSF and two Coup-TFs); one member from subfamily 4 (NR4A); two members from subfamily 5 (FTZ-F1 and HR39) and three 2DBD-NRs (Table 2, S13, S14 Figs). Analysis of the Schistosomatidae species shows that all of them share the same NR complement as $S$. mansoni.

\subsubsection{Order: Opistorchida}

NR complements in three species of Opistorchiidae family were analyzed. They are 1) Clonorchis sinensis (a human liver fluke, its first intermediate hosts are freshwater snails, the second intermediate hosts are freshwater fish and the definitive hosts are humans and other mammals). 2) Opisthorchis viverrini (a human liver fluke, its first intermediate hosts are freshwater snails, the second intermediate hosts are freshwater fish and the definitive hosts are humans and other mammals). 3) O. felineus (a cat liver fluke, its first intermediate hosts are freshwater snails, the second intermediate hosts are freshwater fish and the definitive hosts are humans and other mammals). 

complement in Opistorchida is same as that of Strigeidida, the only difference is that an additional HR96 is present Opistorchida.

\subsubsection{Order: Echinostomida}

\subsubsection{Echinostomatidae family}

NR complement of Echinostoma caproni were mined and analyzed. E. caproni is an intestinal fluke, its first intermediate hosts are freshwater snails and the second intermediate hosts are freshwater snails or frogs, humans can be definitive host. Twenty-two NRs were identified in

\subsubsection{Fasciolidae family}

NR complement of Fasciola hepatica were mined and analyzed. F. hepatica is a liver fluke,

A comparison of Trematoda species, demonstrates that all of them share the same NR complement except that an HR96 is missing in Strigeidida.

\subsection{Class Rhabditophora}

\subsubsection{Order: Macrostomida}


The typical NRs in M. lignano include twenty-one members from subfamily 1 (two TRs,

288

289

290

291

292

293

294

295

296

297

298

299

300

301

302

303

304

305

306

307

308

309

two E78s, sixteen HR96s and NR1a); thirteen members from subfamily 2 (HNF4, two RXRs, two

TR4s, TLL, PNR, DSF, fax1, two NHR236s and two Coup-TFs); four members from subfamily 3

(four ERRs); two members from subfamily 4 (two NR4As); one member from subfamily 5 (HR96);

one member from subfamily 6 (HR6) and two 2DBD-NRs (Table 2, S19 Fig).

An interesting result is that HR96 underwent multiple gene duplications in M. lignano and gave rise to sixteen HR96s (Table 2, S19 Fig). Another important result is that an orthologue of

Drosophila fax-1 and four ERRs are identified in M. lignano, fax-1 and ERR are missing in all of the other analyzed Platyhelminth species. This is the first report that fax-1 and members from subfamily 3 are present in Platyhelminths (Table 2, Fig. 1 and S19 Fig).

\section{Figure 1. ERRs in Platyhelminths}

A) Amino acid sequence alignment of DBD of MlERRs. B) Bayesian phylogenetic tree of MlERRs. The Bayesian tree is constructed with the deduced amino sequences of the DNA binding domain (DBD) with a mix amino acid replacement model + invgamma rates. The trees are started randomly with four simultaneous Markov chains running for 5 million generations. Bayesian posterior probabilities (BPPs) are calculated using a Markov chain Monte Carlo (MCMC) sampling approach implemented in MrBAYES v3.1.1, the PPs values are shown above each branch. Branches under the PPs 0.5 are shown as polytomies. The same data set is also tested by ML method using PHYML (v2.4.4) under LG substitution model (equilibrium frequencies model, proportion of invariable sites: estimated, number of substitution rate categories: 8 , gamma shape parameter: estimated). Support values for the tree were obtained by bootstrapping a 1,000 replicates and bootstrap values above 500 and are indicated below each branch (or after MrBAYES 
BPPs separated by Slash). Star indicates the node obtained by Bayesian inference which is different from that obtained by ML method. Bg: Biomphalaria glabrata, d: Drosophila melanogaster, h: Homo sapiens, Lg: Lottia gigantean, Ml: Macrostomum lignano.

\subsubsection{Order Tricladida}

Schmidtea mediterranea (Dugesidae family), a free-living, freshwater flatworm was

In this study, we re-analyze $S$. mediterranea genome database and twenty-seven NRs were

\section{A novel zinc finger motif (CHC2) exists in DBD of parasitic Platyhelminth NRs} dimerization [64]. 
cysteine residue in the D-Box in CII and it forms a novel zinc finger motif (CHC2). Recently, CHC2 zinc finger motif has been demonstrated to be present in HR96b of Cestoda and other Trematoda species $[66,67]$. In this study, we determined that all SmHR96b orthologues in parasitic Platyhelminths contain this novel CHC2 zinc finger motif (Fig. 2). Thus, $\mathrm{CHC} 2$ type zinc finger motif represents a novel NR CII which has diverged in ancient HR96b in a common ancestor of parasitic Platyhelminths. The function of this novel CHC2 type motif in NRs is unknown. Recent study shows that SmHR96b (named Vitellogenic Factor 1 in $[66,67]$ ) is essential for female sexual development.

\section{Figure 2. A novel zinc finger motif (CHC2) in DBD of parasitic Platyhelminth HR96b}

Amino acid sequence alignment of DBD of the Platyhelminth HR96b shows a novel NR zinc finger motif (CHC2) is present in parasitic Platyhelminth NRs. A histidine residue replaces

the second cysteine residues in the D-Box of zinc finger II forming a novel CHC2 motif. See Fig 1 legend for methods for phylogenetic tree construction. Cs: Clonorchis sinensis, Dl:

Dibothriocephalus latus, Ec: Echinococcus canadensis, Eca: Echinostoma caproni, Eg: Echinococcus granulosus, Em: Echinococcus multilocularis, Fh: Fasciola hepatica, Gs: Gyrodactylus salaris, Hd: Hymenolepis diminuta, Hn: Hymenolepis nana, Ht: Hydatigera taeniaeformis, Lg: Lottia gigantean, Mc: Mesocestoides corti, Ml: Macrostomum lignano, Of: Opisthorchis felineus, Ov: Opisthorchis viverrini, Px: Protopolystoma xenopodis, Sb: Schistosoma bovis, Sc: Schistosoma curassoni, Se: Spirometra erinaceieuropaei, Sh: Schistosoma haematobium, Sj: Schistosoma japonicum, Sm: Schistosoma mansoni, Sma: Schistosoma margrebowiei, Smt: Schistosoma mattheei, Sme: Schmidtea mediterranea, Sr: Schistosoma 
rodhaini, Ss: Schitocephalus solidus, Ta: Taenia asiatica, Tm: Taenia multiceps, Ts: Taenia saginata, Tr: Trichobilharzia regent, Tso: Taenia solium.

\section{Divergent NRs}

Divergent NR refers to a NR which has a typical P-box sequence in DBD but it does not fall into any 'typical' NR groups, for example Platyhelminths NR1a and NR1b with a typical P-box of 'CEGCKGFFRR' belonging to NR subfamily 1 but they do not fit into any groups within the NR1 subfamily. It also refers to a NR which has an atypical P-box sequence in DBD and does not fall into the present NR nomenclature [68].

Divergent NRs exist in various lineages of Platyhelminths. One divergent NR with a typical P-box (CEGCKGFFKR) which is same as that of RXR/TR4/NR4A is found in Rhabditophora $S$. mediterranea and in Cestoda Mesocestoides corti. Most of the Platyhelminth divergent NRs possess an 'atypical' P-box sequence. For example, a NR with a P-box of 'CEPCKVFFKR' is identified in Monogenea G. salaris, a NR with a P-box of 'CEACKAFFQQ' is found in all analyzed species of Cestoda Hymenolepis family, a NR which has a P-box of 'CDSCRAFFEM' exists in Cestoda Taenia family, a NR with a P-box of 'CEACKSFFKR' is found in Cestoda Diphyllobothriidea order and seventeen NRs with various 'atypical' P-box sequences were identified in Rhabditophora M. lignano (S21Fig).

A phylogenetic tree of the divergent NRs including those of Platyhelminths and Mollusca was constructed (S21Fig). Phylogenetic analysis shows no orthologues exist among Rhabditophora M. lignano, Cestoda and Mollusca. The result suggests that these divergent NRs diverged and duplicated independently in different animal lineages. 


\section{Evolution of NR gene in Platyhelminths}

The number of NRs varies in different Platyhelminth species, e.g. 27-62 members are

381

382

\subsection{Subfamily 1}

\subsubsection{E78}

E78 was present as a common ancestor of Platyhelminths. It was retained in Rhabditophora $M$. lignano, Monogenea, Cestoda and Trematoda, but it was missing in Rhabditophora $S$. mediterranea. E78 underwent a duplication in M. lignano and gave a birth of two E78s (MlE78a and MlE78b).

\subsubsection{TR}

One TR is identified in Monogenea; two are identified in Rhabditophora M. lignano and three are identified in $S$. mediterranea; and two are identified in Cestoda and Trematoda, respectively. Our previous study showed that two TR homologues are present in Platyhelminths [12-14, 61]. Phylogenetic analysis suggested that Platyhelminth TR gene duplicated after the split of the trematodes and the turbellarians [61], thus the paralogue of the trematode TR was not present in a turbellarian and in turn the paralogue of turbellarian TR was not present in trematode species.

Phylogenetic analysis in this study shows that all TRs of parasitic Platyhelminths are clustered in a group, two $M$. lignano TRs are clustered in a group and three $S$. mediterranea TRs 
401 are clustered in another group. This result suggests that TRs duplicated independently in $M$. 402 lignano, S. mediterranea and parasitic Platyhelminths. In parasitic Platyhelminth TR groups, trematode TRa (orthologues of Schistosoma TRa, SmTRa) are clustered together with those of

404 Monogenea and Cestoda, but trematode TRb (orthologues of Schistosoma TRb, SmTRb) group 405 only contains trematode TRs. This result suggested that one TR was present in a common ancestor 406 of Platyhelminths and trematode TRa was an ancient TR gene. Phylogenetic analysis shows that 407 the two Cestode M. corti TRs are clustered together in Cestoda TR group, this result suggests that 408 TRs in Cestoda and Trematoda underwent duplication independently (S22 Fig). Since TR 409 duplicated independently in different Platyhelminths lineage, Platyhelminths TRb/TRc are 410 paralogous genes.

\subsubsection{HR96}

Sixteen HR96s are identified in Rhabditophora M. lignano and two are found in $S$. mediterranea; three HR96s are identified in Monogenea, Cestoda and Trematoda, respectively. Phylogenetic analysis shows that Platyhelminthes HR96s are clustered in four different groups: HR96a, HR96b, HR96c and HR96d. HR96a, HR96b and HR96c groups contain only 416 Platyhelminth HR96s, but HR96d group contains both Platyhelminth and Mollusca members. The

417 result suggests that Platyhelminth HR96d is an ancient NR gene (Fig. 3). HR96d group only 418 contains Platyhelminth M. lignano HR96s, but each group of HR96a, HR96b and HR96c contains 419 NR96s from all species of the four classes of Platyhelminths, this result suggests that there were 420 four HR96s in a common ancestor of Platyhelminths and the ancient HR96 gene (HR96d) was lost 421 in Schmidtea mediterranea, Monogenea, Cestoda and Trematoda (Fig. 3). HR96 genes were 422 amplified in M. lignano and each of the four HR96 genes gave birth to a total of sixteen NR96 423 genes. 


\section{Fig. 3. Bayesian phylogenetic tree of Platyhelminth HR96s}

427 glabrata, Cs: Clonorchis sinensis, d: Drosophila melanogaster, Dl: Dibothriocephalus latus, Ec: Taenia solium.

\subsubsection{Divergent NR1a}

442 NR1a was missing in Monogenea G. salaris, Cestoda D. latum, and Hymenolepididae and 443 Taeniidae families. One divergent NR1a is also present in Rhabditophora M. lignano and $S$. 444 mediterranea. Phylogenetic analysis cannot determine if they are orthologues of parasitic 445 Platyhelminth NR1a.

\subsubsection{Divergent NR1b}


Platyhelminths NR1b gene is an orthologue of EgHR3 [45]. It is retained in Rhabditophora $S$.

448

449

450

451

452

453

454

455

456

457

458

459

460

461

462

463

464

465

466

467

468

469

mediterranea, Monogenea $P$. xenopodis and all analyzed tapeworms, it was missing in Rhabditophora M. lignano, Monogenea G. salaris and Trematoda.

\subsection{Subfamily 2}

\subsubsection{HNF4}

HNF4 retained in all analyzed Platyhelminths species.

\subsubsection{RXR}

Two RXRs were identified in Rhabditophora, Monogenea, Cestoda and Trematoda, respectively. Phylogenetic analysis shows that parasitic flatworm RXRs are clustered in two different groups: RXR1 group (Schistosoma RXR1 orthologues) and RXR2 group (Schistosoma RXR2 orthologues). RXR2 group is clustered with RXRs of free-living flatworms, Drosophila, Mollusca and human, this suggests that parasitic Platyhelminth RXR2 is an orthologue of freeliving flatworms, Drosophila, Mollusca and human RXRs. RXR1 group contains only parasitic Platyhelminth RXRs, this suggests that parasitic Platyhelminth RXR2 duplicated after the split of their common ancestor with free-living Platyhelminths. In free-living flatworms, two M. lignano RXRs are clustered in a group while two $S$. mediterranea RXRs are scattered in the phylogenetic tree, this result suggests that RXR duplicated independently in free-living flatworms M. lignano and $S$. mediterranea. The intron position in DBD of RXRs further supports this result (S23 Fig). RXR gene is lost in some of parasitic Platyhelminths after ancient RXR duplicated in a common ancestor of parasitic Platyhelminths: RXR1 was lost in Monogenea G. salaris and Cestoda $D$. latum; RXR2 was lost in Cestoda $S$. erinaceieuropaei; both RXR1 and RXR2 were lost in all the analyzed species of Cestoda Cyclophyllidea order. 


\subsubsection{TR4}

471

TR4 is present in all analyzed Platyhelminth species except two members are present in

472 Rhabditophora M. lignano (MlTR4a and MLTR4b). This suggests that TR4 gene underwent

473 duplication in M. lignano.

474

\subsubsection{TLL}

475

TLL is a conserved NR member, it is present in all analyzed Platyhelminth species.

\subsubsection{PNR}

477

PNR was lost in Cestoda Cyclophyllidea order and Cestoda S. solidus. It is retained in all other analyzed Platyhelminth species.

DSF is absent in Cestoda.

\subsection{7. fax-1}

One fax 1 was identified in Rhabditophora M. lignano, but was missing in all other analyzed Platyhelminths.

\subsubsection{NHR236}

Two NHR236s were present in Rhabditophora M. lignano, one is present in Rhabditophora $S$.

487 Trematoda.

\subsubsection{Coup-TF}

One Coup-TF was identified in Cestoda; two are identified in Rhabditophora, Monogenea and Trematoda, respectively. Phylogenetic analysis shows that parasitic flatworms Coup-TFs are

491 clustered in two different groups: Coup-TFI group (Schistosoma Coup-TFI orthologues) and 492 Coup-TFII group (Schistosoma Coup-TFII orthologues). Coup-TFI group contains members from 
493 all three parasitic classes and it is clustered with that of free-living flatworms, Drosophila,

494 Mollusca and humans. This result suggests that parasitic Platyhelminth Coup-TFI is an ancient

495 Coup-TF gene and it is the orthologue of free-living flatworms, Drosophila, Mollusca and human

496 Coup-TFs. Parasitic Platyhelminth Coup-TFII group contains Monogenea and Trematoda

497 members, this suggests that Coup-TFI duplicated and gave rise to Coup-TFII in a common ancestor

498 of parasitic Platyhelminths. In free-living flatworms, two $M$. lignano Coup-TFs and two $S$.

499 mediterranea Coup-TFs are scattered in the phylogenetic tree, it suggests M. lignano and $S$.

500 mediterranea Coup-TFs duplicated independently; analysis of the intron position in DBD of Coup-

501 TFs further supports this result (S24 Fig). Coup-TFI was missing in Cestoda Hymenolepididae

502 family and in Mesocestoididae (M. corti); Coup-TFII was missing in all analyzed species of

503 Cestoda.

504

\subsection{Subfamily 3}

Four ERRs were identified in Rhabditophora M. lignano. Phylogenetic analysis shows that all four M. lignano ERRs form single group clustered with Drosophila, Mollusca and human ERRs.

507 The result demonstrates that M. lignano ERRs duplicated after a split of Drosophila, Mollusca,

508 Platyhelminths and humans (Fig. 3B). ERR is missing in Rhabditophora S. mediterranea, and in 509 all analyzed parasitic Platyhelminths.

\subsection{Subfamily 4}

Two NR4As were identified in Rhabditophora $M$. lignano and $S$. mediterranea, 513 respectively; one is found in all analyzed parasitic flatworms. Phylogenetic analysis shows that 514 MINR4Aa, SmeNR4Aa and parasitic flatworm NR4A are clustered with Drosophila, Mollusca 515 and human NR4A; it suggests that MINR4Aa, SmeNR4Aa and parasitic flatworm NR4A are 
516 orthologues of Drosophila, Mollusca and human NR4A. MINR4Ab, SmeNR4Ab are scattered in

517 the phylogenetic tree suggesting that they underwent a duplication after a split of M. lignano and

518 S. mediterranea (S25 Fig).

519

520

\subsection{Subfamily 5}

521

\subsection{FTZ-F1}

522

FTZ-F1 is missing in Rhabditophora $M$. lignano, Monogenea G. salaris, Cestoda

Hymenolepididae and Mesocestoididae families. It is retained in all the other analyzed

524 Platyhelminths (S26 Fig).

\subsection{HR39}

526

HR39 is retained in all analyzed Platyhelminths (S26 Fig).

527

528

529

530

531

532

533

534

535

536

537

538

\subsection{Subfamily 6}

NR6 is present in Rhabditophora M. lignano but lost in Rhabditophora S. mediterranea and all analyzed parasitic Platyhelminths.

\subsection{DBD-NR}

Two 2DBD-NRs were identified in Rhabditophora, M. lignano and four were identified in S. mediterranea; three 2DBD-NRs were found in parasitic Platyhelminths including Monogenea, Cestoda and Trematoda, respectively.

A phylogenetic tree of 2DBD-NRs was constructed with the amino acid sequence of the second $\mathrm{DBD}$, because the second $\mathrm{DBD}$ is more conserved than the first DBD. Phylogenetic analysis shows that parasitic Platyhelminth 2DBD-NRs clustered in three groups: 2DBDa (Schistosoma mansoni 2DBD $\alpha$ orthologues), 2DBDb (S. mansoni 2DBD $\beta$ orthologues) and 
2DBDg (S. mansoni 2DBD $\gamma$ orthologues) groups. Parasitic Platyhelminth 2DBD-NRa and 2DBD-

540

541

542

543

544

545

546

547

548

549

550

551

552

553

554

555

556

557

558

559

560

$\mathrm{NRb}$ groups contain members of three classes of parasitic flatworms, but 2DBD-NRg group contains 2DBD-NRs of all the four classes of Platyhelminths and also members of the Mollusca. This result suggests that Parasitic Platyhelminth 2DBD-NRg is an ancient gene, and 2DBD-NRa and 2DBD-NRb were formed by a second round of duplication. In free living Platyhelminths, both Rhabditophora M. lignano 2DBD-NRs are clustered in 2DBD-NRg group, but the four $S$. mediterranea 2DBD-NRs are clustered with different parasitic helminth 2DBD-NR groups. This result suggests that $M$. lignano 2DBD-NR gene underwent duplication after a split of $S$. mediterranea and parasitic Platyhelminths. For the four $S$. mediterranea 2DBD-NRs, one is clustered in parasitic Platyhelminth 2DBD-NRg group, one is clustered in 2DBD-NRb group and two are clustered with 2DBD-NRa group. Since the two $S$. mediterranea (2DBD-NRa1 and 2DBD$\mathrm{NRa} 2$ ) in 2DBDa group form a polytomy, it suggests that 2DBD-NRa underwent another round of duplication and formed two 2DBD-NRs as a common ancestor of $S$. mediterranea and parasitic Platyhelminths and then one 2DBD-NRa was lost in a common ancestor of parasitic Platyhelminths (S27Fig).

The above results suggests that there were at least twenty-four ancient NRs in a common ancestor of Platyhelminths (Fig. 4), there were eight members from subfamily 1 (TR, E78, DHR96a, DHR96b, DHR96c, DHR96d, NR1a and NR1b); ten members from subfamily 2 ( HNF4, RXR2, TR4, TLL, PNR, DSF, dax1, NHR236, Coup-TFI and Coup-TFII); one member from subfamily 3 (ERR); One member from subfamily 4 (NR4A); two members from subfamily 5 (FTZ-F1 and HR39); one member from subfamily 6 (GRF); one member from 2DBD-NRs 
561 (2DBD-NRg). Phylogenetic analysis shows that NR gene gain/loss occurred in different 562 Platyhelminth lineages (Fig. 4).

Figure 4. Summary of NR gene gain and loss in the evolution of Platyhelminths

567 gene gained by gene duplication is shown above the branch of different flatworm lineages and the 568 gene lost is shown under the branches (italic and strikethrough). The number behind flatworm species/families in parentheses indicates the number of NRs identified.

\section{DISCUSSION}

573 Platyhelminths. The results show that NRs in Platyhelminths have orthologues in Deuterostomes, 574 arthropods or both, and the NRs in Platyhelminths diverged into two different evolutionary 575 lineages: 1) Gene duplication and lost; 2) NR gene amplification and divergence.

577 first evolutionary lineage: gene duplication and lost, the events occurred in different flatworm

578 lineages. For example, fax-1 was lost in a common ancestor of $S$. mediterranea and parasitic

579 Platyhelminths, NHR236 was lost in endoparasites (Cestoda and Trematoda), and DSF was lost in

580 Cestoda. In parasitic Platyhelminths, extensive NR gene loss occurred in Cestoda 581 Hymenolepididae and Taeniidae families, e. g. RXR, Coup-TF and FTZ-F1 were retained in most 582 species of Platyhelminths, but they were missing in the cestode families Hymenolepididae and 583 Taeniidae. 
Comparison of NR complement in different Platyhelminth families, NR complement is

585

586

587

588

589

590

591

592

593

594

595

596

597

598

599

600

601

602

603

604

605

most conserved in Trematoda. All species of Trematoda share the same NR complement except that a NHR96 member was lost in Strigeidida (Schistosoma and Trichobilharzia). NR complement in Monogenea and in Cestoda exhibit more differences among different families, in Monogenea, there are eighteen NRs in Gyrodactylidae (G. salaris), but twenty-three NRs are present in Polyopisthocotylea ( $P$. xenopodis). In Cestoda Cyclophyllidea, there are fifteen NRs in Hymenolepididae family, seventeen in Taeniidae family and twenty in Mesocestoididae family.

NR gene duplication occurred in different flatworm lineages. For example, 2DBD-NR underwent a second round of duplication in a common ancestor of $S$. mediterranea and parasitic Platyhelminths, RXR gene duplicated separately in $S$. mediterranea and a common ancestor of parasitic Platyhelminths, and TR duplicated independently in Rhabditophora (S. mediterranea), Cestoda and Trematoda.

NRs in Rhabditophora M. lignano follow another evolutionary lineage: Gene amplification and divergence. In M. lignano, sixty-one NRs are identified including sixteen HR96s and seventeen divergent members. This result suggests that NRs in M. lignano follows an evolutionary lineage with a feature of multiple gene duplication (amplification) and gene divergence. Multiple NR gene duplication was also found in other animals, for example, supplementary NRs (SupNRs) in nematodes and NR1H in Chordate Cephalochordate amphioxus $[69,70]$.

NRs in subfamily 3 and 6 were considered lost in Platyhelminths. In this study, we identified four ERRs and a NR6A in Rhabditophora M. lignano. This is the first known occurrence of NRs in subfamily 3 and 6 in Platyhelminths, but it is still not clear whether NRs from subfamily 3 and 6 are present in other Rhabditophora since the genome data is unavailable. This study also 

study.

shows that divergent NRs are present in different flatworm lineages suggesting that novel NRs were acquired in different flatworms to adapt to the different living environments.

Previously, we isolated a partial cDNA of $S$. mansoni HR96b (SmNR96b) [12], this member has a CHC2 zinc finger motif in the second zinc finger of DBD. In this study, we show that all parasitic Platyhelminth SmHR96b orthologues contain this novel motif. Whether the function of this new type of motif in NRs may change the DNA binding properties awaits further

Acknowledgments: We acknowledge Kevin L. Howe, Bruce J. Bolt, Myriam Shafie, Paul Kersey, and Matthew Berriman for access to WormBase ParaSite - a comprehensive resource for helminth genomics.

\section{REFERENCES}

1. Riutort, M., et al., Evolutionary history of the Tricladida and the Platyhelminthes: an up-to-date phylogenetic and systematic account. Int J Dev Biol, 2012. 56(1-3): p. 5-17.

2. $\quad$ Adell, T., et al., Platyhelminthes, in Evolutionary Developmental Biology of Invertebrates 2: Lophotrochozoa (Spiralia), A. Wanninger, Editor. 2015, Springer Vienna: Vienna. p. 21-40.

3. Norena, C., C. Damborenea, and F. Brusa, Phylum Platyhelminthes. Ecology and General Biology, Vol I: Thorp and Covich's Freshwater Invertebrates, 4th Edition, 2015: p. 181-203.

4. Laudet, V., Evolution of the nuclear receptor superfamily: early diversification from an ancestral orphan receptor. J Mol Endocrinol, 1997. 19(3): p. 207-26.

5. Adams, M.D., et al., The genome sequence of Drosophila melanogaster. Science, 2000. 287(5461): p. 2185-95.

6. Holt, R.A., et al., The genome sequence of the malaria mosquito Anopheles gambiae. Science, 2002. 298(5591): p. 129-49.

7. Bertrand, S., et al., Evolutionary genomics of nuclear receptors: from twenty-five ancestral genes to derived endocrine systems. Mol Biol Evol, 2004. 21(10): p. 1923-37.

8. Sluder, A.E. and C.V. Maina, Nuclear receptors in nematodes: themes and variations. Trends Genet, 2001. 17(4): p. 206-13.

9. Dehal, P., et al., The draft genome of Ciona intestinalis: insights into chordate and vertebrate origins. Science, 2002. 298(5601): p. 2157-67.

10. Zhang, Z., et al., Genomic analysis of the nuclear receptor family: new insights into structure, regulation, and evolution from the rat genome. Genome Res, 2004. 14(4): p. 580-90.

11. Maglich, J.M., et al., Comparison of complete nuclear receptor sets from the human, Caenorhabditis elegans and Drosophila genomes. Genome Biol, 2001. 2(8): p. RESEARCH0029. 
12. Wu, W., et al., Schistosoma mansoni (Platyhelminthes, Trematoda) nuclear receptors: sixteen new members and a novel subfamily. Gene, 2006. 366(2): p. 303-15.

13. Wu, W. and P.T. LoVerde, Nuclear hormone receptors in parasitic helminths. Mol Cell Endocrinol, 2011. 334(1-2): p. 56-66.

14. Wu, W. and P.T. LoVerde, Nuclear hormone receptors in parasitic Platyhelminths. Mol Biochem Parasitol, 2019. 233: p. 111218.

15. Forster, S., et al., Molecular characterisation of a serum-responsive, DAF-12-like nuclear hormone receptor of the fox-tapeworm Echinococcus multilocularis. J Cell Biochem, 2011. 112(6): p. 163042.

16. Wu, W., et al., Evolution of a novel subfamily of nuclear receptors with members that each contain two DNA binding domains. BMC Evol Biol, 2007. 7: p. 27.

17. Howe, K.L., et al., WormBase 2016: expanding to enable helminth genomic research. Nucleic Acids Res, 2016. 44(D1): p. D774-80.

18. Howe, K.L., et al., WormBase ParaSite - a comprehensive resource for helminth genomics. Mol Biochem Parasitol, 2017. 215: p. 2-10.

19. Larkin, M.A., et al., Clustal $W$ and Clustal $X$ version 2.0. Bioinformatics, 2007. 23(21): p. 2947-8.

20. Guindon, S. and O. Gascuel, A simple, fast, and accurate algorithm to estimate large phylogenies by maximum likelihood. Syst Biol, 2003. 52(5): p. 696-704.

21. Guindon, S., et al., New algorithms and methods to estimate maximum-likelihood phylogenies: assessing the performance of PhyML 3.0. Syst Biol, 2010. 59(3): p. 307-21.

22. Wasik, K., et al., Genome and transcriptome of the regeneration-competent flatworm, Macrostomum lignano. Proc Natl Acad Sci U S A, 2015. 112(40): p. 12462-7.

23. Wudarski, J., et al., Efficient transgenesis and annotated genome sequence of the regenerative flatworm model Macrostomum lignano. Nat Commun, 2017. 8(1): p. 2120.

24. Grohme, M.A., et al., The genome of Schmidtea mediterranea and the evolution of core cellular mechanisms. Nature, 2018. 554(7690): p. 56-61.

25. Robb, S.M., et al., SmedGD 2.0: The Schmidtea mediterranea genome database. Genesis, 2015. 53(8): p. 535-46.

26. Robb, S.M., E. Ross, and A. Sanchez Alvarado, SmedGD: the Schmidtea mediterranea genome database. Nucleic Acids Res, 2008. 36(Database issue): p. D599-606.

27. Hahn, C., B. Fromm, and L. Bachmann, Comparative genomics of flatworms (platyhelminthes) reveals shared genomic features of ecto- and endoparastic neodermata. Genome Biol Evol, 2014. 6(5): p. 1105-17.

28. International Helminth Genomes, C., Comparative genomics of the major parasitic worms. Nat Genet, 2019. 51(1): p. 163-174.

29. Tsai, I.J., et al., The genomes of four tapeworm species reveal adaptations to parasitism. Nature, 2013. 496(7443): p. 57-63.

30. Maldonado, L.L., et al., The Echinococcus canadensis (G7) genome: a key knowledge of parasitic platyhelminth human diseases. BMC Genomics, 2017. 18(1): p. 204.

31. Zheng, H., et al., The genome of the hydatid tapeworm Echinococcus granulosus. Nat Genet, 2013. 45(10): p. 1168-75.

32. Wang, S., et al., Comparative genomics reveals adaptive evolution of Asian tapeworm in switching to a new intermediate host. Nat Commun, 2016. 7: p. 12845.

33. Li, W., et al., The genome of tapeworm Taenia multiceps sheds light on understanding parasitic mechanism and control of coenurosis disease. DNA Res, 2018. 25(5): p. 499-510.

34. Bennett, H.M., et al., The genome of the sparganosis tapeworm Spirometra erinaceieuropaei isolated from the biopsy of a migrating brain lesion. Genome Biol, 2014. 15(11): p. 510. 
35. Wang, X., et al., The draft genome of the carcinogenic human liver fluke Clonorchis sinensis. Genome Biol, 2011. 12(10): p. R107.

36. Ershov, N.I., et al., New insights from Opisthorchis felineus genome: update on genomics of the epidemiologically important liver flukes. BMC Genomics, 2019. 20(1): p. 399.

37. Young, N.D., et al., The Opisthorchis viverrini genome provides insights into life in the bile duct. Nat Commun, 2014. 5: p. 4378.

38. Cwiklinski, K., et al., The Fasciola hepatica genome: gene duplication and polymorphism reveals adaptation to the host environment and the capacity for rapid evolution. Genome Biol, 2015. 16: p. 71.

39. McNulty, S.N., et al., Genomes of Fasciola hepatica from the Americas Reveal Colonization with Neorickettsia Endobacteria Related to the Agents of Potomac Horse and Human Sennetsu Fevers. PLoS Genet, 2017. 13(1): p. e1006537.

40. Oey, H., et al., Whole-genome sequence of the bovine blood fluke Schistosoma bovis supports interspecific hybridization with S. haematobium. PLoS Pathog, 2019. 15(1): p. e1007513.

41. Young, N.D., et al., Whole-genome sequence of Schistosoma haematobium. Nat Genet, 2012. 44(2): p. 221-5.

42. Schistosoma japonicum Genome, S. and C. Functional Analysis, The Schistosoma japonicum genome reveals features of host-parasite interplay. Nature, 2009. 460(7253): p. 345-51.

43. Berriman, M., et al., The genome of the blood fluke Schistosoma mansoni. Nature, 2009. 460(7253): p. 352-8.

44. Wu, W., et al., Identification and characterization of a nuclear receptor subfamily I member in the Platyhelminth Schistosoma mansoni (SmNR1). FEBS J, 2007. 274(2): p. 390-405.

45. Yang, M., et al., Cloning and characterization of an Echinococcus granulosus ecdysteroid hormone nuclear receptor HR3-like gene. Parasite, 2017. 24: p. 36.

46. Tharp, M.E., J.J. Collins, 3rd, and P.A. Newmark, A lophotrochozoan-specific nuclear hormone receptor is required for reproductive system development in the planarian. Dev Biol, 2014. 396(1): p. $150-7$.

47. Bertin, B., et al., The monomeric orphan nuclear receptor Schistosoma mansoni Ftz-F1 dimerizes specifically and functionally with the schistosome RXR homologue, SmRXR1. Biochem Biophys Res Commun, 2005. 327(4): p. 1072-82.

48. Bertin, B., et al., Unique functional properties of a member of the Fushi Tarazu-Factor 1 family from Schistosoma mansoni. Biochem J, 2004. 382(Pt 1): p. 337-51.

49. De Mendonca, R.L., et al., A functionally conserved member of the FTZ-F1 nuclear receptor family from Schistosoma mansoni. Eur J Biochem, 2002. 269(22): p. 5700-11.

50. de Mendonca, R.L., et al., Structural and functional divergence of a nuclear receptor of the RXR family from the trematode parasite Schistosoma mansoni. Eur J Biochem, 2000. 267(11): p. 320819.

51. Escriva, $\mathrm{H}_{\text {., }}$ et al., Ligand binding was acquired during evolution of nuclear receptors. Proc Natl Acad Sci U S A, 1997. 94(13): p. 6803-8.

52. Freebern, W.J., E.G. Niles, and P.T. LoVerde, $R X R-2$, a member of the retinoid $x$ receptor family in Schistosoma mansoni. Gene, 1999. 233(1-2): p. 33-8.

53. Freebern, W.J., et al., Identification of a cDNA encoding a retinoid $X$ receptor homologue from Schistosoma mansoni. Evidence for a role in female-specific gene expression. J Biol Chem, 1999. 274(8): p. 4577-85.

54. Hu, R., E.G. Niles, and P.T. LoVerde, DNA binding and transactivation properties of the Schistosoma mansoni constitutive androstane receptor homologue. Mol Biochem Parasitol, 2006. 150(2): p. 174-85. 
55. Hu, R., et al., SmTR2/4, a Schistosoma mansoni homologue of TR2/TR4 orphan nuclear receptor. Int J Parasitol, 2006. 36(10-11): p. 1113-22.

56. Hu, R., et al., Isolation and characterization of Schistosoma mansoni constitutive androstane receptor. Mol Biochem Parasitol, 2006. 148(1): p. 31-43.

57. Lu, C., E.G. Niles, and P.T. LoVerde, Characterization of the DNA-binding properties and the transactivation activity of Schistosoma mansoni nuclear receptor fushi tarazu-factor 1alpha (SmFTZ-F1alpha). Mol Biochem Parasitol, 2006. 150(1): p. 72-82.

58. Lu, C., et al., Identification and characterization of a novel fushi tarazu factor 1 (FTZ-F1) nuclear receptor in Schistosoma mansoni. Mol Biochem Parasitol, 2006. 150(1): p. 25-36.

59. Wendt, G., et al., A single-cell RNA-seq atlas of Schistosoma mansoni identifies a key regulator of blood feeding. Science, 2020. 369(6511): p. 1644-1649.

60. Wu, W. and P.T. Loverde, Schistosoma mansoni: identification of SmNR4A, a member of nuclear receptor subfamily 4. Exp Parasitol, 2008. 120(2): p. 208-13.

61. Wu, W., E.G. Niles, and P.T. LoVerde, Thyroid hormone receptor orthologues from invertebrate species with emphasis on Schistosoma mansoni. BMC Evol Biol, 2007. 7: p. 150.

62. Wu, W., E.Y. Tak, and P.T. LoVerde, Schistosoma mansoni: SmE78, a nuclear receptor orthologue of Drosophila ecdysone-induced protein 78. Exp Parasitol, 2008. 119(2): p. 313-8.

63. Ladurner, P., et al., A new model organism among the lower Bilateria and the use of digital microscopy in taxonomy of meiobenthic Platyhelminthes: Macrostomum lignano, $n . s p$ (Rhabditophora, Macrostomorpha). Journal of Zoological Systematics and Evolutionary Research, 2005. 43(2): p. 114-126.

64. Luisi, B.F., et al., Crystallographic analysis of the interaction of the glucocorticoid receptor with DNA. Nature, 1991. 352(6335): p. 497-505.

65. Lee, M.S., et al., Structure of the retinoid X receptor alpha DNA binding domain: a helix required for homodimeric DNA binding. Science, 1993. 260(5111): p. 1117-21.

66. Wang, J., R. Chen, and J.J. Collins, 3rd, Systematically improved in vitro culture conditions reveal new insights into the reproductive biology of the human parasite Schistosoma mansoni. PLoS Biol, 2019. 17(5): p. e3000254.

67. Wang, J. and J.J. Collins, 3rd, Identification of new markers for the Schistosoma mansoni vitelline lineage. Int J Parasitol, 2016. 46(7): p. 405-10.

68. Nuclear Receptors Nomenclature Committee, A unified nomenclature system for the nuclear receptor superfamily. Cell, 1999. 97(2): p. 161-3.

69. Lecroisey, C., V. Laudet, and M. Schubert, The cephalochordate amphioxus: a key to reveal the secrets of nuclear receptor evolution. Brief Funct Genomics, 2012. 11(2): p. 156-66.

70. Gobert, G.N., et al., Tissue specific profiling of females of Schistosoma japonicum by integrated laser microdissection microscopy and microarray analysis. PLoS Negl Trop Dis, 2009. 3(6): p. e469.

\section{Supplemental FIGURE LEGENDS}

\section{S1 Fig. Bayesian phylogenetic tree of Protopolystoma xenopodis NRs}

The Bayesian tree was constructed with the deduced amino sequences of the DNA binding domain (DBD) with a mix amino acid replacement model + invgamma rates. The trees were started randomly with four simultaneous Markov chains running for 5 million generations. Bayesian 
posterior probabilities (BPPs) are calculated using a Markov chain Monte Carlo (MCMC) sampling approach implemented in MrBAYES v3.1.1, the PPs values are shown above each branch. Branches under the PPs 0.5 are shown as polytomies. The same data set is also tested by ML method using PHYML (v2.4.4) under LG substitution model (equilibrium frequencies model, proportion of invariable sites: estimated, number of substitution rate categories: 8 , gamma shape parameter: estimated). Support values for the tree are obtained by bootstrapping a 1,000 replicates and bootstrap values above 500 and are indicated below each branch (or after MrBAYES BPPs separated by Slash). Star indicates the node obtained by Bayesian inference which is different from that obtained by ML method. Bg: Biomphalaria glabrata, Cg: Crassostrea gigas, d: Drosophila melanogaster, h: Homo sapiens, Lg: Lottia gigantean, Px: Protopolystoma xenopodis, Sm: Schistosoma mansoni. Red highlighted NRs show P. xenopodis NRs.

\section{S2 Fig. Bayesian phylogenetic tree of NR1 DBD sequences}

Methods for construction of phylogenetic trees see S1 fig legend. Bg: Biomphalaria glabrata, d: Drosophila melanogaster, Dl: Dibothriocephalus latus, Eca: Echinostoma caproni, Eg: Echinococcus granulosus, Fh: Fasciola hepatica, h: Homo sapiens, Hd: Hymenolepis diminuta, Ht: Hydatigera taeniaeformis, Lg: Lottia gigantean, Mc: Mesocestoides corti, Ml: Macrostomum lignano, Ov: Opisthorchis viverrini, Px: Protopolystoma xenopodis, Se: Spirometra erinaceieuropaei, Sm: Schistosoma mansoni, Sme: Schmidtea mediterranea, Ss: Schitocephalus solidus, Tm: Taenia multiceps, Tr: Trichobilharzia regent. Red highlighted NRs show Platyhelminths NRs. 
Methods for construction of phylogenetic trees see S1 fig legend. Bg: Biomphalaria

799

800

801

802

803

804

805

806

807

808

809

810

811

812

813

814

815

816

817

818

819

glabrata, d: Drosophila melanogaster, Eg: Echinococcus granulosus, h: Homo sapiens, Lg: Lottia

gigantean, Sme: Schmidtea mediterranea. Red highlighted NRs show Platyhelminths NRs.

\section{S4 Fig. Bayesian phylogenetic tree of NR1 LBD}

Methods for construction of phylogenetic trees see S1 fig legend. Bg: Biomphalaria glabrata, d: Drosophila melanogaster, Eg: Echinococcus granulosus, h: Homo sapiens, Lg: Lottia gigantean, Sme: Schmidtea mediterranea. Red highlighted NRs show Platyhelminths NRs.

\section{S5 Fig. Bayesian phylogenetic tree Gyrodactylus salaris NRs}

Methods for construction of phylogenetic trees see S1 fig legend. Bg: Biomphalaria glabrata, Cg: Crassostrea gigas, d: Drosophila melanogaster, Gs: Gyrodactylus salaris, h: Homo sapiens, Lg: Lottia gigantean, Px: Protopolystoma xenopodis, Sm: Schistosoma mansoni. Red highlighted NRs show G. salaris NRs.

\section{S6 Fig. Bayesian phylogenetic tree of Hymenolepis microstoma NRs}

The phylogenetic tree of H. microstoma NRs represents all analyzed Hymenolepis species because of the highly conserved DBD sequences in these species. Methods for construction of phylogenetic trees see S1 fig legend. Bg: Biomphalaria glabrata, Cg: Crassostrea gigas, d: Drosophila melanogaster, h: Homo sapiens, Hm: Hymenolepis microstoma, Lg: Lottia gigantean, Px: Protopolystoma xenopodis, Sm: Schistosoma mansoni. Red highlighted NRs show $H$. microstoma NRs.

\section{S7 Fig. Bayesian phylogenetic tree of Mesocestoides corti NRs}

Methods for construction of phylogenetic trees see S1 fig legend. Bg: Biomphalaria glabrata, Cg: Crassostrea gigas, d: Drosophila melanogaster, h: Homo sapiens, Lg: Lottia 
820 gigantean, Mc: Mesocestoides corti, Px: Protopolystoma xenopodis, Sm: Schistosoma mansoni.

821 Red highlighted NRs show $M$. corti NRs.

822 S8 Fig. Bayesian phylogenetic tree of Echinococcus multilocularis NRs mansoni. Red highlighted NRs show E. multilocularis NRs.

\section{S9 Fig. Bayesian phylogenetic tree of Hydatigera taeniaeformis NRs}

\section{S10 Fig. Bayesian phylogenetic tree of Taenia saginata NRs}

837 S11 Fig. Bayesian phylogenetic tree of Schitocephalus solidus NRs 
Methods for construction of phylogenetic trees see S1 fig legend. Bg: Biomphalaria

844

845

846

847

848

849

850

851

852

853

854

855

856

857

858

859

860

861

862

863

864

865

glabrata, Cg: Crassostrea gigas, d: Drosophila melanogaster, h: Homo sapiens, Lg: Lottia

gigantean, Px: Protopolystoma xenopodis, Se: Spirometra erinaceieuropaei, Sm: Schistosoma

mansoni. DBD of E78 sequence is partial, not used for construction of the tree. Red highlighted

NRs show S. erinaceieuropaei NRs.

\section{S13 Fig. Bayesian phylogenetic tree of Schistosoma haematobium NRs}

The phylogenetic tree of $S$ haematobium NRs represents all analyzed Schistosoma species

because of the highly conserved DBD sequences in these species. Methods for construction of phylogenetic trees see S1 fig legend. Bg: Biomphalaria glabrata, Cg: Crassostrea gigas, d:

Drosophila melanogaster, h: Homo sapiens, Lg: Lottia gigantean, Px: Protopolystoma xenopodis, Sh: Schistosoma haematobium, Sm: Schistosoma mansoni. Red highlighted NRs show S. haematobium NRs.

\section{S14 Fig. Bayesian phylogenetic tree of Trichobilharzia regent NRs}

Methods for construction of phylogenetic trees see S1 fig legend. Bg: Biomphalaria glabrata, Cg: Crassostrea gigas, d: Drosophila melanogaster, h: Homo sapiens, Lg: Lottia gigantean, Px: Protopolystoma xenopodis, Sm: Schistosoma mansoni, Tr: Trichobilharzia regent. Red highlighted NRs show T. regent NRs.

\section{S15 Fig. Bayesian phylogenetic tree of Clonorchis sinensis NRs}

Methods for construction of phylogenetic trees see S1 fig legend. Bg: Biomphalaria glabrata, Cg: Crassostrea gigas, Cs: Clonorchis sinensis, d: Drosophila melanogaster, h: Homo sapiens, Lg: Lottia gigantean, Px: Protopolystoma xenopodis, Sm: Schistosoma mansoni. Red highlighted NRs show $C$. sinensis NRs.

S16 Fig. Bayesian phylogenetic tree of Opisthorchis viverrini NRs 
871 NRs.

872 S17 Fig. Bayesian phylogenetic tree of Echinostoma caproni NRs 
Methods for construction of phylogenetic trees see S1 fig legend. Bg: Biomphalaria

890

891

892

893

894

895

896

897

898

899

900

901

902

903

904

905

906

907

908

909

910

911

glabrata, Cg: Crassostrea gigas, d: Drosophila melanogaster, h: Homo sapiens, Lg: Lottia gigantean, Px: Protopolystoma xenopodis, Sm: Schistosoma mansoni, Sme: Schmidtea mediterranea. Red highlighted NRs show $S$. mediterranea NRs.

\section{S21 Fig. Bayesian phylogenetic tree of Platyhelminth divergent NRs}

Methods for construction of phylogenetic trees see S1 fig legend. Bg: Biomphalaria glabrata, d: Drosophila melanogaster, Dl: Dibothriocephalus latus, Ec: Echinococcus Canadensis, Eg: Echinococcus granulosus, Em: Echinococcus multilocularis, Gs: Gyrodactylus salaris, h: Homo sapiens, Hd: Hymenolepis diminuta, Hm: of H. microstoma, Ht: Hydatigera taeniaeformis, Mc: Mesocestoides corti, Ml: Macrostomum lignano, Sme: Schmidtea mediterranea, Ta: Taenia asiatica, Tm: Taenia multiceps, Ts: Taenia saginata, Tr: Trichobilharzia regent, Tso: Taenia solium.

\section{S22 Fig. Bayesian phylogenetic tree of Platyhelminth TRs}

Methods for construction of phylogenetic trees see S1 fig legend. Bg: Biomphalaria glabrata, Cs: Clonorchis sinensis, d: Drosophila melanogaster, Dl: Dibothriocephalus latus, Ec: Echinococcus Canadensis, Eca: Echinostoma caproni, Eg: Echinococcus granulosus, Em: Echinococcus multilocularis, Fh: Fasciola hepatica, Gs: Gyrodactylus salaris, h: Homo sapiens, Hd: Hymenolepis diminuta, Hm: of H. microstoma, Hn: Hymenolepis nana, Ht: Hydatigera taeniaeformis, Lg: Lottia gigantean, Mc: Mesocestoides corti, Ml: Macrostomum lignano, Of: Opisthorchis felineus, Ov: Opisthorchis viverrini, Px: Protopolystoma xenopodis, Sb: Schistosoma bovis, Sc: Schistosoma curassoni, Se: Spirometra erinaceieuropaei, Sh: Schistosoma haematobium, Sj: Schistosoma japonicum, Sm: Schistosoma mansoni, Sma: Schistosoma margrebowiei, Smt: Schistosoma mattheei, Sme: Schmidtea mediterranea, Sr: Schistosoma 
912 rodhaini, Ss: Schitocephalus solidus, Ta: Taenia asiatica, Tm: Taenia multiceps, Ts: Taenia

913 saginata, Tr: Trichobilharzia regent, Tso: Taenia solium.

914 S23 Fig. Bayesian phylogenetic tree of Platyhelminth RXR

915 A) Bayesian phylogenetic tree of Platyhelminth RXR. Methods for construction of 916 phylogenetic trees see S1 fig legend. Bg: Biomphalaria glabrata, Cs: Clonorchis sinensis, d:

917 Drosophila melanogaster, Dl: Dibothriocephalus latus, Ec: Echinococcus Canadensis, Eca:

918 Echinostoma caproni, Eg: Echinococcus granulosus, Em: Echinococcus multilocularis, Fh:

919 Fasciola hepatica, Gs: Gyrodactylus salaris, h: Homo sapiens, Lg: Lottia gigantean, Ml:

920 Macrostomum lignano, Of: Opisthorchis felineus, Ov: Opisthorchis viverrini, Px: Protopolystoma

921 xenopodis, Sb: Schistosoma bovis, Sc: Schistosoma curassoni, Se: Spirometra erinaceieuropaei,

922 Sh: Schistosoma haematobium, Sj: Schistosoma japonicum, Sm: Schistosoma mansoni, Sma:

923 Schistosoma margrebowiei, Smt: Schistosoma mattheei, Sme: Schmidtea mediterranea, Sr:

924 Schistosoma rodhaini, Ss: Schitocephalus solidus, Tr: Trichobilharzia regent. B) Sequence

925 alignment shows the intron position (red color $>$ ) in DBD of RXRs.

926 S24 Fig. Bayesian phylogenetic tree of Platyhelminth Coup-TF

A) Bayesian phylogenetic tree of Platyhelminth Coup-TF. Methods for construction of 928 phylogenetic trees see S1 fig legend. Bg: Biomphalaria glabrata, Cs: Clonorchis sinensis, d: 929 Drosophila melanogaster, Dl: Dibothriocephalus latus, Ec: Echinococcus Canadensis, Eca: Echinostoma caproni, Eg: Echinococcus granulosus, Em: Echinococcus multilocularis, Fh:

931 Fasciola hepatica, Gs: Gyrodactylus salaris, h: Homo sapiens, Lg: Lottia gigantean, Ml: 932 Macrostomum lignano, Of: Opisthorchis felineus, Ov: Opisthorchis viverrini, Px: Protopolystoma 933 xenopodis, Sb: Schistosoma bovis, Sc: Schistosoma curassoni, Se: Spirometra erinaceieuropaei, 934 Sh: Schistosoma haematobium, Sj: Schistosoma japonicum, Sm: Schistosoma mansoni, Sma: 
Schistosoma margrebowiei, Smt: Schistosoma mattheei, Sme: Schmidtea mediterranea, Sr: Schistosoma rodhaini, Ss: Schitocephalus solidus, Ta: Taenia asiatica, Tm: Taenia multiceps, Ts: Taenia saginata, Tr: Trichobilharzia regent, Tso: Taenia solium. B) Sequence alignment shows the intron position (red color $>$ ) in DBD of Coup-TFs.

\section{S25 Fig. Bayesian phylogenetic tree of Platyhelminth NR4A}

Methods for construction of phylogenetic trees see S1 fig legend. Bg: Biomphalaria glabrata, d: Drosophila melanogaster, Gs: Gyrodactylus salaris, h: Homo sapiens, Hd: Hymenolepis diminuta, Lg: Lottia gigantean, Mc: Mesocestoides corti, Ml: Macrostomum lignano, Sm: Schistosoma mansoni, Sme: Schmidtea mediterranea.

\section{S26 Fig. Bayesian phylogenetic tree of subfamily 5}

Methods for construction of phylogenetic trees see S1 fig legend. Bg: Biomphalaria glabrata, d: Drosophila melanogaster, Dl: Dibothriocephalus latus, Ec: Echinococcus Canadensis, Eca: Echinostoma caproni, Em: Echinococcus multilocularis, Fh: Fasciola hepatica, Gs: Gyrodactylus salaris, h: Homo sapiens, Hn: Hymenolepis nana, Lg: Lottia gigantean, Mc: Mesocestoides corti, Ml: Macrostomum lignano, Of: Opisthorchis felineus, Ov: Opisthorchis viverrini, Px: Protopolystoma xenopodis, Se: Spirometra erinaceieuropaei, Sj: Schistosoma japonicum, Sm: Schistosoma mansoni, Smt: Schistosoma mattheei, Sme: Schmidtea mediterranea, Tr: Trichobilharzia regent, Tso: Taenia solium.

\section{S27 Fig. Bayesian phylogenetic tree of Platyhelminth 2DBD}

Methods for construction of phylogenetic trees see S1 fig legend. Bg: Biomphalaria glabrata, Cs: Clonorchis sinensis, d: Drosophila melanogaster, D1: Dibothriocephalus latus, Em: Echinococcus multilocularis, Fh: Fasciola hepatica, Gs: Gyrodactylus salaris, h: Homo sapiens, Hd: Hymenolepis diminuta, Hm: H. microstoma, Lg: Lottia gigantean, Mc: Mesocestoides corti, 
bioRxiv preprint doi: https://doi.org/10.1101/2021.04.14.439782; this version posted April 14,2021 . The copyright holder for this preprint (which

was not certified by peer review) is the author/funder, who has granted bioRxiv a license to display the preprint in perpetuity. It is made available under aCC-BY 4.0 International license.

958 Ml: Macrostomum lignano, Px: Protopolystoma xenopodis, Se: Spirometra erinaceieuropaei, Sm:

959 Schistosoma mansoni, Sme: Schmidtea mediterranea, Ss: Schitocephalus solidus, Tr:

960 Trichobilharzia regent.

961 


\section{P-Box}

BgERR LgERR hERRa hERRb dERR MIERR1 MIERR2 MIERR3 MIERR4 $\mathrm{hERb}$ hERa LgER hGR hPR hAR BgER hMrepow

CLVCG-DVASGYHYGVSSCEACKA CLVCG-DIASGYHYGVSSCEACKA CLVCG-DVASGYHYGVA. CEA AKA CLVCG-DIASGYHYGVASCEACKA CLVCG-DVASGFHYGVAS CRVCH-RRA SGHHYGALT CEA CKA CSICF-RKATGHHYGAVTCE A KKAFFKRTVHSCVTYF-CPVQRSGSRCPVPKSSRKRHCPACRWVRCLEARM CQVCLNFPQTGRHYGAVSCEA AKAFFKRTVQQCLVYQ-CLAS---VLCTELSSK-RRSCPKCRFQRCLKAGM CSICG-APPSGVHYGATICEA AKAFFKRTVHSCLTYS-CIRPGNPGQCPVHGNR-RRQCAACRWRRCLTVGM CAVCS-DYASGYHYGVWSCEGCKAFFKRS IQGHNDYI-CPATN---QCTIDKNR-RKSCQACRLRKCYEVGM CAVCN-DYASGYHYGVWOCEGCKAFFKRSIQGHNDYM-CPATN---QCTIDKNR-RKSCQACRLRKCYEVGM CQVCN-DNASGFHYGVWOCEGCKAFFKRSIQGPVDYV-CPATN---NCTIDKHR-RKSCQACRLRKCYEVGM CQVCN-DNASGFHYGVW CEGCKAFFKRS IQGPVDYI-CPATN---TCTIDKHR-RKSCQACRLRRCYEVGM CLVCS-DEASGCHYGVLTCGSCKVFFKRAVEGQHNYL-CAGRN---DCIIDKIR-RKNCPACRYRKCLQAGM

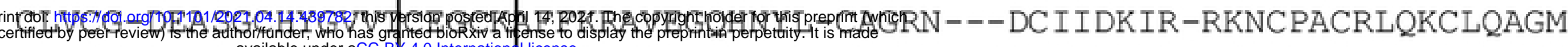

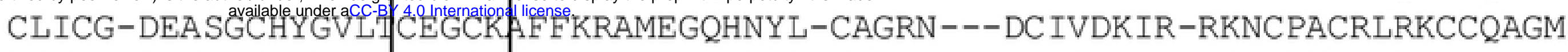
CLICG-DEASGCHYGALICEGCKAFFKRAAEGKQKYL-CASRN---DCTIDKFR-RKNCPSCRLRKCYEAGM

0.2

B

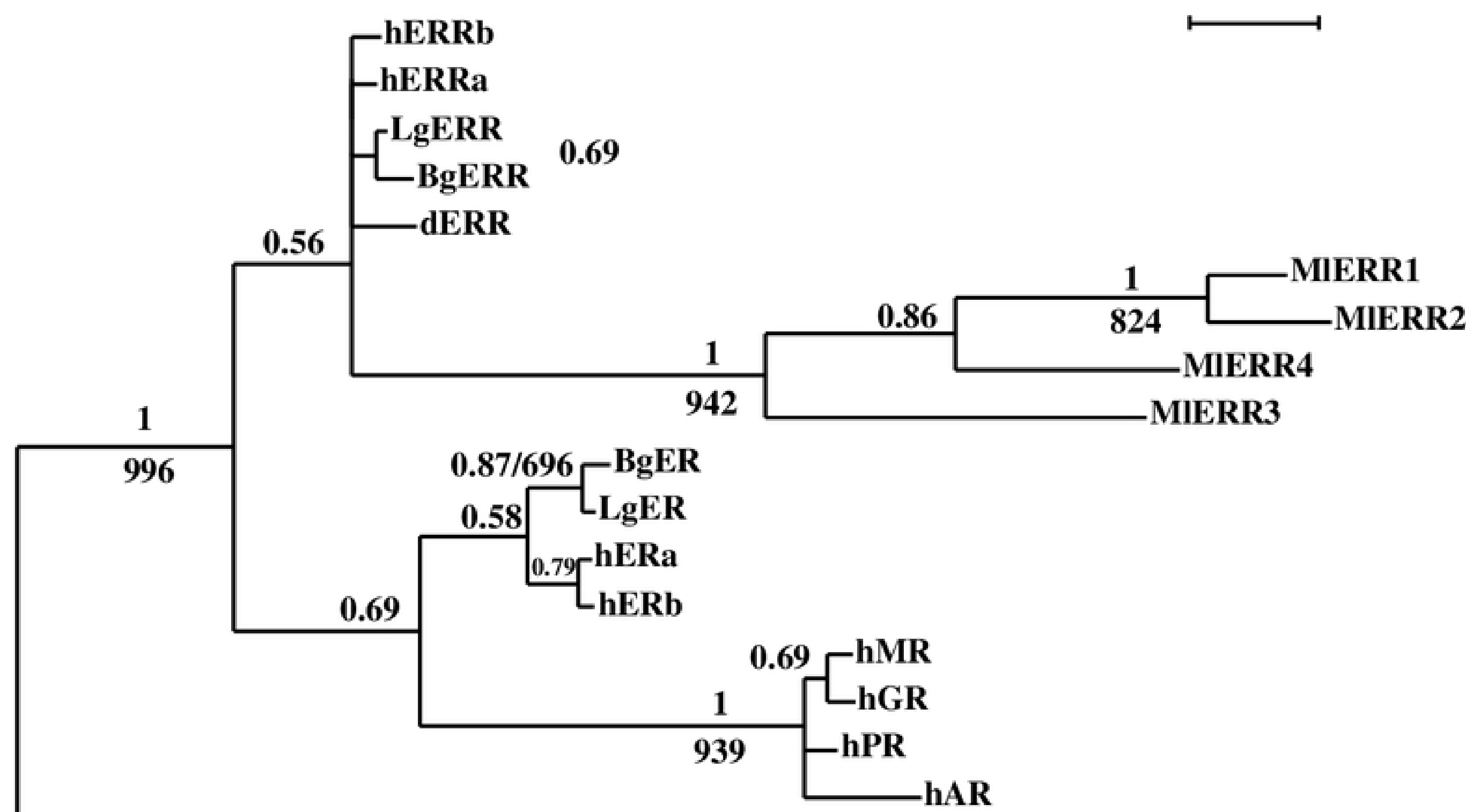

0.79 hRev-erbA 555 Lev-erbB

dE75

Fig. 1

Figure 1 ERR 
OvNR96b OfNR96b EcaHR96b FhNR96b SmNR96b ShNR96b SjNR96b ScNR96B SmaHR96b SmtNR96b SrNR96b

TrNR96b

HdHR96b HmHR96b

HaHR96b

McHR96b

EmHR96b

EcHR96b

EgHR96B

HtHR $96 b$

TaHR96b

TmHR96b

TsoNR96b

D1NR96b

SsHR96b

SeHR96b

CsNR96b

GsHR96b

PxHR96b

- SmeHR96-3

M1HR96-12

M1HR96-13

M1HR96-14
P-BOX

CKVCGDRAVNHNFGQLTC CKVCGDRAVNHNFGQLTC CKVCNDRAVNHNFGQLTC CKVCNDRAVNHNFGQLTC CKVCGDRAVNHNFGQLTC CKVCGDRAVNHNFGQLTC CKVCGDRAVNHNFGQLTC CKVCGDRAVNHNFGQLTC CKVCGDRAVNHNFGQLTC CKVCGDRAVNHNFGQLTC CKVCGDRAVNHNFGQLTC

CRVCGDRAVNHNFGQLTC CRVCGDRAVNHNFGQLTC CRVCGDRAVNHNFGQLTC CRVCGDRAVNHNFGQLTC CRVCGDRAVNHNFGQLTC CRVCGDRAVNHNFGQLTC CRVCGDRAVNHNFGQLTC CRVCGDRAVNHNFGQLTC CRVCGDRAVNHNFGQLTC CRVCGDRAVNHNFGQLTC CRVCGDRAVNHNFGQLTC

CKVCGDRAVNHNFGQLTC CVVCGEPASGYNFDRLTC CKVCGDRAVNHNFGQLTC CKVCGDRAVNHNFGQLTC CKVCGDKAVNHNFGQLTC CKVCFDKAVNQNFGVLSC CRVCGDLAATFNFGQICC CLVCGCKAENYNFGVISC CLVCGAKAENYNFGVISC ESCKA

ESCKA

BSCKA ESCKA BSCKA ESCKA ESCKA ESCKA ESCKA
ESCKA FFRRNAHKLLPSLLDRTFECRSDVD-----MTTVQYSLSLSRSKYLVELT ESCKA FFRRNAHKLLPSLLDRTFECRSDID-----MTTVQYSLSLSRSKYLVELT

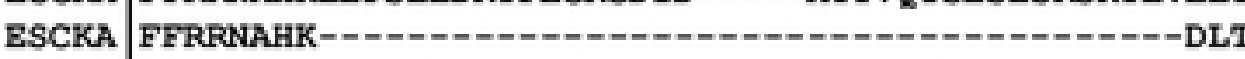
ESCKA FFRRNAHKGLSTLMVTRMLRTNDTS----SLIYESNRTAHIKSRGLVELT ESCKA FFRRNAHKGLSTLMVTRMLRTNDTS----SLIYEPNRTAHIKSRGLVELT ESCKA FFRRNAHKGLSTLLETRMLRTNDTS----SLLYESNRTSHIRSRGLVELT ESCKA F FRRNAHKGLSTLMVTRMLRTNDTS----SLIYEPNRTAHIKSRGLVELT ESCKA FERRNAHKGLSTLMVTRMLRTNDTS----SLIYEPNRTAHIKSRGLVELT ESCKA FFRRNAHKGL STLMVTRMLRTNDTS----SLIYEPNRTAHIKSRGLVELT ESCKA FFRRNAHKGLSTLMVTRMLRTNDTS----SLIYESNRTAHIKSRGLVELT F FRRNAHKALPSI SAVLQSSGTKSI------TMEVYIKAIQYGLRDL ESCKA FFRRNAHKALPSI SAVLQSSGTKSI--------TMEVYIKAIQHGLPDLT ESCKA FFRRNAHKALPSINAALQSCAPGMP-------TSDAYVRAVRLGLSELT ESCKA FFRRNAHKALPSI SAALQSSDCAT $=-\cdots-=-T T D A Y L Q A L Q R G L R D L T$ ESCKA FRRNAHKALP SI SAALQSSDCAT---------TTDAYLQALQRGLRDLT ESCKA TFRRNAHKALPSISAALOSSDCAT---------TTDAYLOALORGLRDLT ESCKA FFRRNAHKALPSI IAALRSSGDAT--------TTDAYLQALQRGLRDLT ESCKA FFRRNAHKALPSI IAALRSSGDAT--------TTDAYLOALRRGLRDLT ESCKA FFRRNAHKALPSI IAALRSSGDAT--------TTDAYLQALRRGLRDLT ESCKA FFRRNAHKALPSI IAALRSSGDAT---------TTDAYLQALRRGLRDLT
D-BOX

CTSKTGE H EITPSTRRECPACRLKKCFLVGM CTSKTGE H EITPSTRRECPACRLKKCFLVGM CTLKSGE H EITPTTRRECPACRLIKKCFRVGM CTLKAGE H EITPTTRRECPACRLKKCFRVGM CTAKSGE H VITPTTRRECPSCRLKOCFRVGM CTAKSGE H VITPTTRRECPSCRLKQCFRVGM CTAKSGE H VITPTTRRECPSCRLKOCFRVGM CTAKSGE H VITPTTRRECPSCRLKQCFRVGM CTAKSGE H VITPTTRRECPSCRLKQCFRVGM CTAKSGE H VITPTTRRECPSCRLKQCFRVGM CTAKSGE H VITPTTRRECPSCRLKQCFRVGM CTAKSGE H I ITPTTRRECPSCRLKOCFRVGM CTSKSGE CTSKSGE CTSKSGE CTSKSGE CTSKSGE CTSKSGE CTSKSGE CTSKSGE CTSKSGE CTSKSGE CTSKSGE $\mathrm{H}$

\section{CTSKTNG}

CTSKVTG

CSNGTDL

CSHGDGN

CSFRRGG CSFQHAR
VVSPSTRRECPACRLKRCFLIGM VVSPSTRRECPACRLKRCFLIGM VVSPSTRRECPACRLKRCFLIGM VVSPSTRRECPACRLKRCFLIGM VVSPSTRRECPACRLKRCELIGM VVSPSTRRECPACRLKRCFLIGM VVSPSTRRECPACRLKRCFLIGM VVSPSTRRECPACRLKRCFLIGM VVSPSTRRECPACRLKRCFLIGM VVSPSTRRECPACRLKRCFLIGM VVSPSTRRECPACRLKRCFLIGM VVSPTTRRECPACRLKRCFLIGM VVSPTTRRECPACRLKRCFLIGM IVSPTTRRECPACRLIKRCFLIGM EITPSTRRECPACRLKKCFLVGM YISPSTRRECPACRLKRCFFVGM VISPTTRRECPACRLIRCFVVGM SVTSSTRKQCPSCRLKKCLQVGM LITVTTRRTCRACRFEKCLAVGM EVSLATRKKCPGCRLAKCFAVGM EVTLATRKKCPGCRLARCFRVGM

\section{Zinc Finger I}

\section{Zinc Finger II}

Fig. 2 


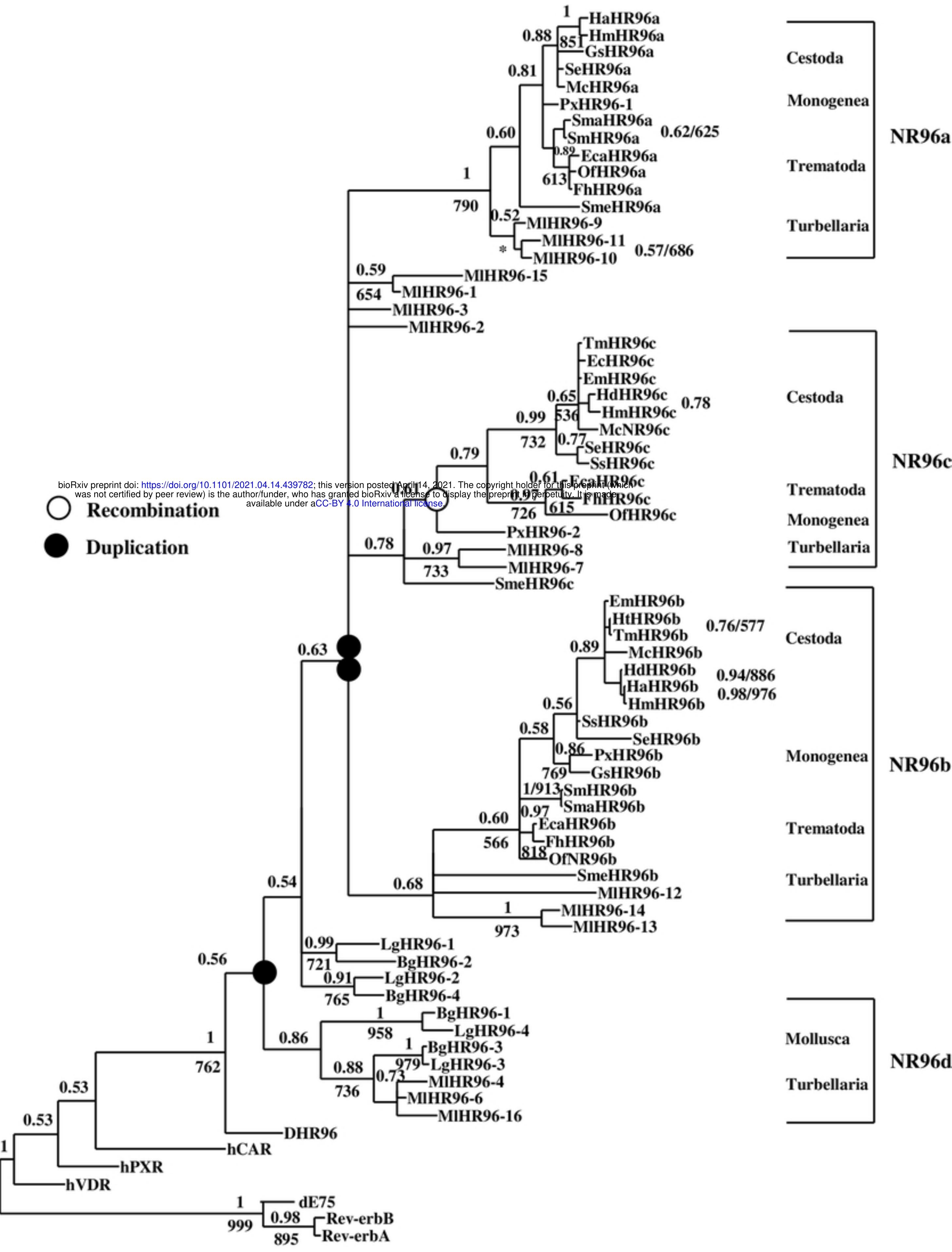

\section{Figure 3}

Figure 3 HR96 


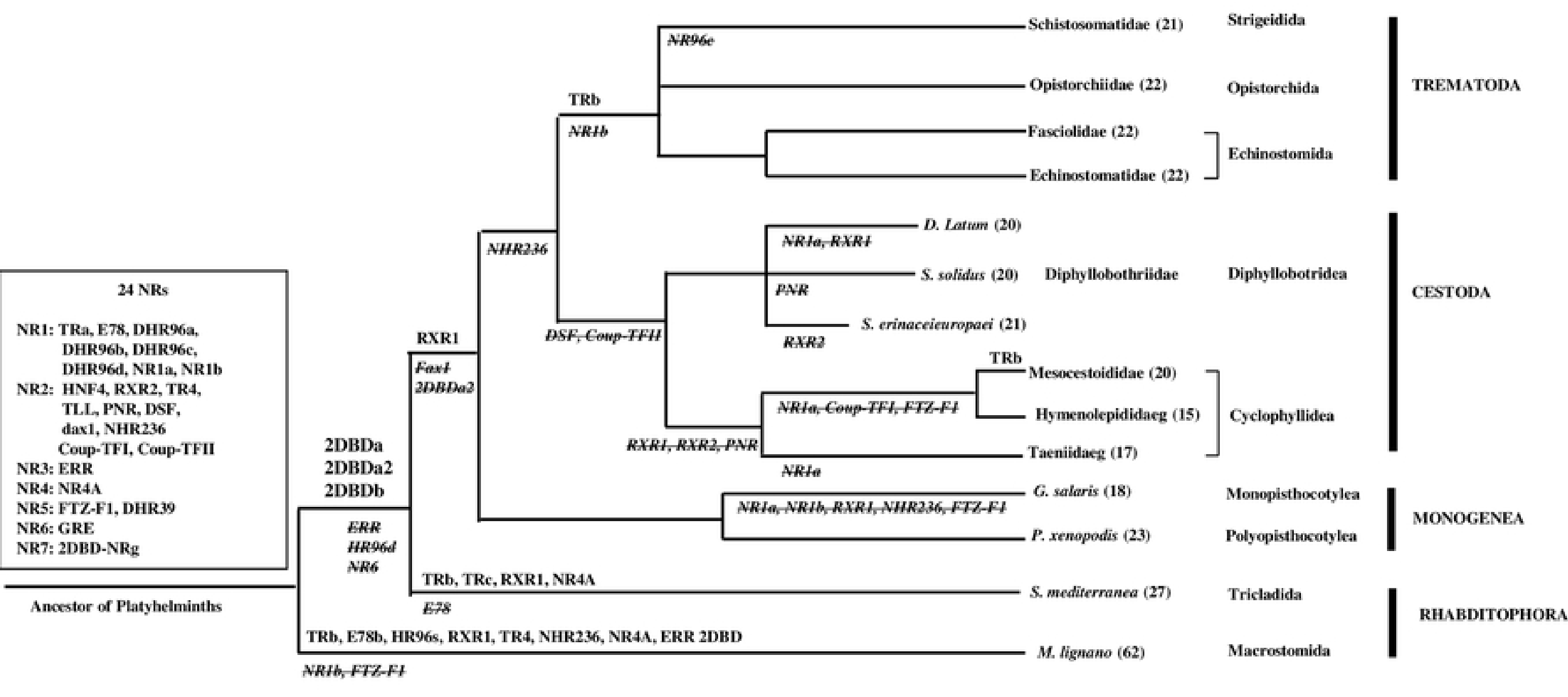

\section{Figure 4}

Figure 4 Evolution of NRs 